\title{
Iron overload induced death of osteoblasts in vitro: involvement of the mitochondrial apoptotic pathway
}

\author{
Qing Tian ${ }^{1}$, Shilei Wu ${ }^{1}$, Zhipeng Dai ${ }^{2}$, Jingjing Yang ${ }^{3}$, Jin Zheng ${ }^{4}$, Qixin Zheng ${ }^{\text {Corresp., }}{ }^{1}$, Yong Liu ${ }^{\text {Corresp. } 1}$ \\ 1 Department of Orthopedics, Union Hospital, Tongji Medical College, Huazhong University of Science and Technology, Wuhan, Republic of China \\ 2 Department of Orthopedics, Henan Provincial People's Hospital, Zhengzhou, Republic of China \\ 3 Department of Child Health, Changzhou Maternal and Child Health Care Hospital, Changzhou, Republic of China \\ 4 Department of Neurology, Union Hospital, Tongji Medical College, Huazhong University of Science and Technology, Wuhan, Republic of China \\ Corresponding Authors: Qixin Zheng, Yong Liu \\ Email address: zheng-qx@163.com, 573846007@qq.com
}

Background. Iron overload isrecognized as a new pathogenfor osteoporosis. Various studies demonstrated that iron overload could induce apoptosis in osteoblasts and osteoporosis in vivo. However, the exact molecular mechanisms involved in the iron overload-mediated induction of apoptosis in osteoblasts has not been explored.

Purpose. In this study, we attempted to determine whether the mitochondrial apoptotic pathway is involved in iron-induced osteoblastic cell death and to investigate the beneficial effect of $\mathrm{N}$-acetylcysteine (NAC) in iron-induced cytotoxicity.

Methods. The MC3T3-E1 osteoblastic cell line was treated with various concentrations of ferric ion in the absence or presence of NAC, and intracellular iron, cell viability, reactive oxygen species, functionand morphology changes of mitochondria and mitochondrial apoptosis related key indicators were detected by commercial kits. In addition, to further explain potential mechanisms underlying iron overload-related osteoporosis, we also assessed cell viability, apoptosis, and osteogenic differentiation potential in bone marrow-derived mesenchymal stemcells(MSCs) by commercial kits.

Results. Ferric ion demonstrated concentration-dependent cytotoxic effects on osteoblasts. After incubation with iron, an elevation of intracelluar labile iron levels and a concomitant over-generation of reactive oxygen species (ROS) were detected by flow cytometry in osteoblasts. Nox4 (NADPH oxidase 4), an important ROS producer, was also evaluated by western blot. Apoptosis, which was evaluated by Annexin V/propidium iodide staining, Hoechst 33258 staining, and the activation of caspase-3, was detected after exposure to iron. Iron contributed to the permeabilizatio of mitochondria, leading to the release of cytochrome $\mathrm{C}$ (cyto $\mathrm{C}$ ), which, in turn, induced mitochondrial apoptosis in osteoblasts via activation of Caspase-3, up-regulation of Bax, and down-regulation of Bcl-2. NAC could reverse ironmediated mitochondrial dysfunction and blocked the apoptotic events through inhibit the generation of ROS. In addition, iron could significantly promote apoptosis and suppress osteogenic differentiation and mineralization i n bone marrow-derived MSCs.

Conclusions. These findings firstly demonstrate that the mitochondrial apoptotic pathway involved in iron-induced osteoblast apoptosis. NAC could relieved the oxidative stress and shielded osteoblasts from apoptosis casused by iron-overload. We also reveal that iron overload in bone marrow-derived MSCs results in increased apoptosis and the impairment of osteogenesis and mineralization. 
1 Iron Overload Induced Death of Osteoblasts In Vitro: Involvement of

4 Qing Tian ${ }^{1 *}$,Shilei $\mathrm{Wu}^{1 *}$, Zhipeng $\mathrm{Dai}^{2}$, Jingjing Yang ${ }^{3}$, Jin Zheng ${ }^{4}$, Qixin Zheng ${ }^{1}$, Yong Liu ${ }^{1}$

$5 \quad{ }^{1}$ Department of Orthopedics, Union Hospital, Tongji Medical College, Huazhong University of

6 Science and Technology, Wuhan, Hubei, China

$7 \quad 2$ Department of Orthopedics, Henan Provincial People's Hospital, Zhengzhou, Henan, China

$8{ }^{3}$ Department of Child Health, Changzhou Maternal and Child Health Care Hospital, Changzhou, 9 Jiangsu, China

${ }^{4}$ Department of Neurology, Union Hospital, Tongji Medical College, Huazhong University of

11 Science and Technology, Wuhan, Hubei, China

$12{ }^{*}$ These authors contributed equally to this work.

\section{Corresponding author}

15 Yong Liu (liuyongxiehexiqu@163.com)

16 Qixin Zheng (zheng-qx@163.com)

17 Department of Orthopedics, Union Hospital, Tongji Medical College, Huazhong University of 
18 Science and Technology, Wuhan, Hubei, China

19

20 


\section{Abstract}

22 Background. Iron overload is recognized as a new pathogen for osteoporosis. Various studies

23 demonstrated that iron overload could induce apoptosis in osteoblasts and osteoporosis in vivo.

24 However, the exact molecular mechanisms involved in the iron overload-mediated induction of

25 apoptosis in osteoblasts has not been explored.

26 Purpose. In this study, we attempted to determine whether the mitochondrial apoptotic pathway

27 is involved in iron-induced osteoblastic cell death and to investigate the beneficial effect of N-

28 acetyl-cysteine (NAC) in iron-induced cytotoxicity.

29 Methods. The MC3T3-E1 osteoblastic cell line was treated with various concentrations of ferric

30 ion in the absence or presence of NAC, and intracellular iron, cell viability, reactive oxygen

31 species, function and morphology changes of mitochondria and mitochondrial apoptosis related

32 key indicators were detected by commercial kits. In addition, to further explain potential

33 mechanisms underlying iron overload-related osteoporosis, we also assessed cell viability,

34 apoptosis, and osteogenic differentiation potential in bone marrow-derived mesenchymal

35 stem cells (MSCs) by commercial kits.

36 Results. Ferric ion demonstrated concentration-dependent cytotoxic effects on osteoblasts. After

37 incubation with iron, an elevation of intracelluar labile iron levels and a concomitant overgeneration of reactive oxygen species (ROS) were detected by flow cytometry in osteoblasts. Nox4 (NADPH oxidase 4), an important ROS producer, was also evaluated by western blot. 
40 Apoptosis, which was evaluated by Annexin V/propidium iodide staining, Hoechst 33258

41 staining, and the activation of caspase-3, was detected after exposure to iron. Iron contributed to

42 the permeabilizatio of mitochondria, leading to the release of cytochrome $\mathrm{C}$ (cyto $\mathrm{C}$ ), which, in

43 turn, induced mitochondrial apoptosis in osteoblasts via activation of Caspase-3, up-regulation of

44 Bax, and down-regulation of Bcl-2. NAC could reverse iron-mediated mitochondrial dysfunction

45 and blocked the apoptotic events through inhibit the generation of ROS. In addition, iron could

46 significantly promote apoptosis and suppress osteogenic differentiation and mineralization in

47 bone marrow-derived MSCs.

48 Conclusions. These findings firstly demonstrate that the mitochondrial apoptotic pathway 49 involved in iron-induced osteoblast apoptosis. NAC could relieved the oxidative stress and

50 shielded osteoblasts from apoptosis casused by iron-overload. We also reveal that iron overload

51 in bone marrow-derived MSCs results in increased apoptosis and the impairment of osteogenesis

52 and mineralization. 


\section{Introduction}

55

56

5

58

59

60

61

62

63

64

Iron is an essential element for several cellular and metabolic processes. However, this transition metal also catalyzes the formation of damaging free radicals, leading to the oxidative injury of cellular components. Osteoporosis and fractures occur frequently in patients with disorders associated with iron overload such as thalassemia and hemochromatosis (Vogiatzi MG et al., 2006; Vogiatzi MG et al., 2009; Kim BJ et al., 2012; Wong P et al., 2014). Evidence from numerous studies indicates that iron overload directly exerts detrimental effects on bone metabolism (Yang $Q$ et al., 2011; Tsay J et al., 2010). Excessive iron accumulation in osteoblast triggers apoptosis which may play essential roles in osteoporosis (Messer JG et al., 2009; Doyard M et al., 2012). However, the mechanism by which iron induces apoptosis is not fully understood. Therefore, the elucidation of the mechanisms underlying apoptosis and development of therapeutic strategies to block apoptosis in osteoblasts are crucial for treating iron-overload induced osteoporosis.

Apoptosis occurs via two major pathways: the death receptor pathway and mitochondrial pathway. The death receptor pathway is mainly initiated by the ligation of death receptors such as tumor necrosis factor (TNF) and Fas/CD95, in which the recruited caspase 8 acts as a trigger for the activation of caspase 3 and apoptosis (Fuchs Y et al., 2011). The mitochondrial pathway, known as another important apoptotic pathway, is activated by various stimuli that induce the dissipation of the mitochondrial membrane and the release of apoptotic factors such as cyto $c$ (Kroemer G et al., 2007; Green DR et al., 2014). After cyto c is released into the cytosol, its 
74 initiates the formation of cytochrome c /Apaf-1/ Caspase-9 complex (termed the apoptosome),

75 which causes the activation of Caspase-3, subsequently executing cell apoptosis (Tait $S W$ et al.,

76 2013). However, the specific apoptotic pathway by which iron induces apoptosis in osteoblasts

77 has not been reported.

78 Studies have confirmed that redox-active iron in mitochondria is capable of directly

79 catalyzing the formation of deterimental free radicals via Fenton chemistry (Lill R., 2009; Dixon

80 SJ et al., 2014). Both iron-dependent ROS and high concentrations of labile iron are thought to

81 contribute to mitochondrial ultrastructural damage (Pietrangelo A et al., 2016). Therefore, we

82 hypothesize that iron-induced osteoblast apoptosis may be mediated by the mitochondrial

83 apoptotic pathway. Furthermore, we explored the role of the mitochondrial pathway in iron-

84 overload induced osteoblast apoptosis by examining mitochondrial function and apoptosis

85 related key molecules. In additon, in our present study, the protection of NAC on iron overload-

86 induced osteoblasts apoptosis has been investigate.

88 Materials \& Methods

\section{Materials}

N-acetyl-cysteine (NAC), Ferric ammonium citrate (FAC), 2',7'-dichlorodihy-

drofluorescein diacetate (H2DCF-DA), calcein-AM, Hoechst33258, 4',6-diamidino-2-

phenylindol (DAPI), penicillin, streptomycin, leupeptin, pepstatin A, deaprotinin, 
93

94

phenylmethylsulfonylfluoride (PMSF), and 4-(2-hydroxyethyl)-1-piperazineethane sulfonic acid (HEPES) were purchased from Sigma (USA). Primary antibodies against cytochrome c, bcl-2, bax, cleaved Caspase-3, Glyceraldehyde-3-Phosphate Dehydrogenase (GAPDH) and beta-actin ( $\beta$-actin) were purchased from Abcam (UK). AnnexinV-FITC/PI kit was obtained from KeyGen Biotech (China). Fetal bovine serum and alpha-modified Eagle's medium (a-MEM) were purchased from Gibco (USA). Cell Mitochondria Isolation Kit, Enhanced Chemiluminescence detection kit, Western and immunoprecipitation (IP) Cell Lysis Kit, Bicinchoninic Acid Protein (BCA) Protein Assay Kit, and 5,5',6,6'-tetrachloro-1,1',3,3'-

tetraethylbenzimidazolcarbocyanineiodide (JC-1) were purchased from Beyotime (China). Cell Counting Kit-8 (CCK-8) assay kit was purchased from DojinDo (Japan).

\section{Cell cultures and treatment}

MC3T3-E1 osteoblasts (obtained from American Type Culture Collection) were cultured in alpha-modified Eagle's medium supplemented with 10\% fetal bovine serum (FBS) (Gibco H, Invitrogen, Grand Island, NY, USA), $50 \mathrm{U} / \mathrm{ml}$ penicillin, and $50 \mathrm{mg} / \mathrm{ml}$ streptomycin. The medium was changed thrice one week. Cells were cultured to $80-90 \%$ confluence, harvested, and seeded at $1 \times 10^{4} \mathrm{cell} / \mathrm{cm}^{2}$ in 96 - and 6 -well plates.

FAC, which functions as an iron donor, was used to simulate iron overload conditions in vitro (Doyard M et al., 2012; Zarjou A et al., 2010). We incubated MC3T3-E1 cells with various concentrations of FAC: 25, 50, 100, and $200 \mu \mathrm{M}$. Control groups were treated with PBS. After exposure to FAC in the absence or presence of NAC ( $1 \mathrm{mM})$, all samples were collected 
113 subsequent analyses by flow cytometry, Western Blot, confocal microscopy, and fluorescence

114 microscopy.

Primary bone marrow-derived MSCs were isolated from Sprague-Dawley rats (100-120 g, obtained from the Animal Center of Tong Ji Medical College, Huazhong University of Science and Technology) as previously described (Meng $J$ et al., 2016). The isolated cells were cultured in 55- $\mathrm{m}^{2}$ dishes in Dulbecco's modified Eagle's medium (HyClone, USA) with 10\% (v/v) fetal bovine serum (FBS) and $100 \mu \mathrm{g} / \mathrm{mL}$ streptomycin and penicillin(Beyotime-biotechnology, China) at $37^{\circ} \mathrm{C}, 5 \% \mathrm{CO}_{2}$ atmosphere. The growth medium was changed every 2 days. Primary bone marrow-derived MSCs were grown to confluence and used from passages 3 to 6 throughout the 122 following experiments.

123

\section{Measurement of cell viability}

The cell counting kit-8 was applied to determine viability of osteoblastic cells and bone marrow-derived MSCs as described previously(Ding F et al., 2012; Cai XY et al., 2015). After exposure to FAC $(25-200 \mu \mathrm{M})$ for 24 and $120 \mathrm{~h}$, the mixture solution containing medium $(90 \mu \mathrm{l})$ and CCK-8 reactant $(10 \mu \mathrm{l})$ was added to each well of a 96- well plate. Then, the sample was incubated at $37^{\circ} \mathrm{C}$ for $2 \mathrm{~h}$ in the dark. Finally, the absorbance at $450 \mathrm{~nm}$ was analyzed in a spectrophotometer (Thermo, USA).

\section{Assay of the intracellular labile iron level by flow cytometry and fluorescence microscopy}

The labile iron pool (LIP), which refers to the level of intracellular redox-active and 
132 133

chelatable iron, has been implicated in cellular damage by catalyzing excess-production of deterimental free radical. The intracellular LIP was measured by flow cytometry following calcein staining. Briefly, after treatment with FAC $(0-200 \mu \mathrm{M})$ for $120 \mathrm{~h}$, the osteoblasts were collected and resuspended in PBS, and subsequently treated with $0.25 \mu \mathrm{M}$ calcein-AM in dark at $37^{\circ} \mathrm{C}$ for $30 \mathrm{~min}$ (Tenopoulou M et al., 2007; Glickstein H et al., 2005; Kaur D et al., 2008).

Next, osteoblasts were rinsed twice with serum-free a-MEM, gently resuspended in the medium, and immediately analyzed by flow cytometry using CellQuest analysis software (BD Biosciences, USA). Meanwhile, in order to evaluate the change in intracellular labile iron levels in situ, all samples were additionally monitored under a fluorescence microscope.

\section{Evaluation of intracellular reactive oxygen species}

Levels of ROS in osteoblasts were determined with H2DCF-DA, a fluorescent dye, which could be rapidly oxidized into the highly fluorescent compound DCF in the presence of ROS (Ma KG et al., 2013). Following treatment with FAC, the osteoblasts were collected and washed with PBS, subsequently resuspended in serum-free media, and finally treated with $20 \mu \mathrm{M}$ H2DCF-DA in dark at $37^{\circ} \mathrm{C}$ for 20 min (Ding $F$ et al., 2012). After incubation, MC3T3-E1 cells were rinsed with serum-free a-MEM thrice, and subsequently the mean fluorescence intensity (MFI) was evaluated with a FACSCalibur flow cytometer (BD, USA).

\section{Evaluation of apoptosis by Annexin V-FITC/PI staining}

$$
\text { After treatment as described above, osteoblasts and bone marrow-derived MSCs from each }
$$


151

152

153

154

155

156

157

158

159

160

161

162

163

164

165

166

167

168

169

sample were stained using the Annexin V-FITC/PI kit, as described previously (Ding F et al., 2012; Cai XY et al., 2015). Then, flow cytometry was accomplished by flow cytometric analysis (BD, USA). The sample was additionally visualized under a confocal microscope (OLYMPUS FV1000, Japan). The Annexin V+/PI- osteoblasts were considered early apoptotic cells (Henry CM et al., 2013). The Annexin V+/PI+ osteoblasts were considered late apoptotic cells (Henry CM et al., 2013) .

\section{Evaluation of apoptosis-related morphologic changes in osteoblasts}

To verify apoptosis, ding (Ding F et al., 2012; Cai XY et al., 2015) outlined a procedure for ascertaining apoptosis, through the application of Hoechst 33258 dye at $0.5 \mu \mathrm{g} / \mathrm{mL}$ concentrate, to clarify the fractured and compressed apoptotic nuclei. Following the application of the dye, the nuclei were stained for one hour at $37^{\circ} \mathrm{C}$ with all light sources omitted. The nuclei were then given two washes with PBS, so as to remove the Hoechst 33258. Utilising a fluorescence microscope manufactured by Carl Zeiss, the morphology of osteoblast apoptosis was pictured with UV excitation at $350 \mathrm{~nm}$, then analysed.

\section{Measurement of mitochondrial membrane potential (MMP)}

The collapsed MMP in osteoblasts was detected by flow cytometry following JC-1 dye staining (Beyotime-biotechnology, China). Briefly, after treatment as described above, the harvested MC3T3-E1 cells were resuspended in a staining solution, which were prepared by admixing serum-free a-MEM (500 $\mu \mathrm{L})$ and JC-1 staining fluid (500 $\mu \mathrm{L})$. Subsequently, 
170

171

172

173

174

175

176

177

178

179

180

181

182

osteoblasts were incubated with the staining solution for $20 \mathrm{~min}$ at $37^{\circ} \mathrm{C}$ in the dark. After incubation, osteoblasts were washed thrice with the JC-1 staining buffer (Beyotimebiotechnology, China), and resuspended in $500 \mu \mathrm{L}$ of cell culture medium prior to analysis by flow cytometry. Finally, the ratio of red fluorescence intensity to green fluorescence intensity was calculated, and the results were used to evaluate the change in MMP for each sample(Ding $F$ et al., 2012).

$$
\text { In order to evaluate the change in MMP in situ, osteoblasts were loaded with JC-1 dye after }
$$
treatment with FAC, as described above (Ding F et al., 2012). Aggregated JC-1 (red fluorescence) and monomeric JC-1 (green fluorescence) levels were observed using laser scanning confocal microscopy (Olympus FV1000, Japan).

\section{Western blot analysis}

After treatment with FAC, as described above, osteoblasts were collected and homogenized in lysis buffer. The details of the Western blotting procedures have been described previously (Ding F et al., 2012). The monoclonal antibodies used were as follows: anti-cleaved Caspase-3 (1:2000), anti-Bax (1:1000), anti-Bcl-2 $(1: 1000)$, and anti-Cytochrome c (1:1000). GAPDH and beta-actin were utilized as internal controls.

\section{Induction of osteogenic differentiation of bone marrow-derived MSCs and Alizarin Red} staining

\footnotetext{
To detect the iron effect on osteogenic differentiation, Cyagen ${ }^{\circledR}$ osteogenesis differentiation
} 
medium (Cyagen Biosciences., China) were used following the manufacturer's protocol (Zhu Y et al., 2012). Briefly, primary bone marrow-derived MSCs were cultured in osteogenesis differentiation media $(2 \mathrm{mmol} / \mathrm{L} \beta$-glycerol-phosphate, $50 \mu \mathrm{mol} / \mathrm{L}$ ascorbic acid, $0.1 \mu \mathrm{mol} / \mathrm{L}$ dexamethasone) alone or in the presence of $\mathrm{FAC}$ for 14 days. After induction, osteogenesis was eveluated by staining MSCs with Alizarin Red S reagent (Cyagen Biosciences., China) as protocol described.

\section{Evaluation of the deposition of calcium}

Primary bone marrow-derived MSCs were subcultured in 6-well plates in growth medium (Dulbecco's modified Eagle's medium, 10\% fetal bovine serum (FBS) and $100 \mathrm{lg} / \mathrm{mL}$ streptomycin and penicillin). After primary bone marrow-derived MSCs had reached approximately $80 \%$ confluence, FAC was added to the osteogenesis differentiation medium. To quantify the iron effect on the matrix calcification, Alizarin red S staining were used following the manufacturer's protocol (Zhang $X$ et al., 2010). Positive red staining represents calcium deposits of on the differentiated MSCs. For quantification of staining, $100 \mathrm{mM}$ cetypyridinium chloride was added to each plate and used to extracte Alizarin red S, and the sample was incubated at room temperature for $3 \mathrm{~h}$ (Malladi $P$ et al., 2006). Finally, the absorbance of the extracted Alizarin red S at $570 \mathrm{~nm}$ was analyzed in a spectrophotometer (Thermo, USA).

\section{Measurement of Alkaline phosphatase activity (ALP)}

$$
\text { Primary bone marrow-derived MSCs treated on 6-well plates were rinsed with PBS thrice, }
$$


208 lysed in RIPA solution (Beyotime-biotechnology, China), and finally centrifuged to remove

209 cellular debris at $4^{\circ} \mathrm{C}$. Next, ALP activity in the samples was measured by p-nitrophenyl

210 phosphate method using a Alkaline Phosphatase Assay Kit (Beyotime-biotechnology, China) as

211 previous report (Lyu Z et al., 2014).

\section{Statistical analysis}

$213 \quad$ All data were expressed as means \pm standard

214 deviation(SD).Statistical analyses were performed with SPSS software (version 18.0) for

215 Windows software. In order to analyze statistical differences between samples, we used one-

216 way analysis of variance (ANOVA) with least significant difference (LSD). P $<0.05$ was

217 considered statistically significant.

\section{Results}

\section{Influences of iron on osteoblastic cell viability} iron in a dose-dependent manner. However, after a 24-h exposure to FAC, no statistically

224 significant difference was observed between the viability of osteoblasts at the various

225 concentrations tested[Fig.1]. These results imply that iron may undergo accumulation in

226 osteoblasts, which may elicit cytotoxic effects in these cells. 
227

228

229

230

231

232

233

234

235

236

237

\title{
Increase in intracellular labile iron levels due to iron overload in osteoblasts
}

\author{
Labile iron pool, known as free and chelatable iron, is the major potentially toxic form in
} iron-overload related diseases (Brissot $P$ et al., 2011). To evaluate changes of LIP in osteoblasts, a fluorescent iron-sensitive probe, calcein-AM was used. When calcein-AM permeates into the osteoblast and binds the intracellular labile iron, its fluorescence is quenched enabling evaluation of the intracellular labile iron levels by via measurement of the decrease in calcein-AM fluorescence (Tenopoulou M et al., 2007; Glickstein H et al., 2005; Kaur D et al., 2008). After incubation with FAC $(25-200 \mu \mathrm{M})$ for $120 \mathrm{~h}$, the mean fluorescence intensity of calcein-AM decreased in a dose-dependent manner, which indicated a significant increase in the intracellular labile iron levels within the osteoblasts [Fig. 2].

\section{Impacts of iron on ROS level and expression of NADPH oxidase 4 (Nox4) in osteoblasts}

To determine whether the over-production of ROS plays a pivotal role in iron-induced apoptosis, we used the specific fluorescence dye, H2DCF-DA, which detected intracellular ROS formation. According to the results of H2DCF-DA staining, a concentration-dependent increase in intracellular ROS production was observed in osteoblasts exposed to various concentrations of FAC (25-200 $\mu \mathrm{M})$ for $120 \mathrm{~h}$ [Fig. 3] ; the ROS levels were found to be 1.82- ,4.00- , 7.75-, and 10.55-fold higher after treatment of osteoblast with 25, 50, 100, and $200 \mu \mathrm{M} \mathrm{FAC}$, respectively

[Fig. 3(B)]. NADPH oxidase is one of the most important ROS producers within the cell (Sahoo S et al., 2016). In our study, we determined that FAC affects expression of Nox4, which mainly mediates the clinical phenotype in bone loss-related diseases (Manolagas SC et al., 2010; 
247 Schröder $K$ et al., 2015). Our results indicate that FAC $(25-200 \mu \mathrm{M})$ upregulates Nox4 in

248 osteoblasts.

249 Effects of iron on apoptosis in osteoblasts

In order to assess whether iron mediated toxicity in osteoblasts is related to the activation of apoptosis, a following investigation was made. Annexin V/PI apoptosis assay kit were used to

252

253

254

255

256

257

258

259

260

261

262

263

264

265

evlaute the apoptosis rate of osteoblasts. Annexin V+/PI- osteoblasts and Annexin+/PI+

osteoblasts were considered apoptotic cells. Following the application of FAC for $120 \mathrm{~h}$ at 0,25 , 50, 100 and $200 \mu \mathrm{M}$, apoptosis was seen to raise from $4.41 \%$ to $56.72 \%$, as illustrated by [Fig.

4(B)]. Furthermore, compared to control group (FAC $0 \mu \mathrm{M})$, there was a mainly raised amount of late apoptosis after FAC $(200 \mu \mathrm{M})$ expouse in [Fig. 4(A)], with [Fig. 4(C)] also suggestive of a similar impact in osteoblasts.

Phase-contrast microscopy and fluorescence microscopy were also utilised to observe the morphology of osteoblasts and the degree of apoptosis resulting from iron exposure. The resluts of nuclear-staining with Hoechst indicated that, compared to $0 \mu \mathrm{M} \mathrm{FAC} \mathrm{(control),} \mathrm{the} \mathrm{proportion}$ of osteoblasts with condensed or fragmented nuclei markedly increased in the FAC treatment group [Fig. 5(A)]. Phase-contrast microscopy indicated that osteoblasts incubated with FAC (200 $\mu \mathrm{M})$ for $120 \mathrm{~h}$ revealed apoptosis-related morphology, characterized by cell shrinkage, rounding and floating in 6-well plates [Fig. 5(B)].

\section{Involvement of cleaved Caspase-3, cytochrome c, Bax and Bcl-2 in iron-induced apoptosis}


In order to confirm that the mitochondrial pathway associated with iron treatment, we

267

268

269

270

271

272

273

274

275

276

277

278

279

280

281

282

283

284

285

detected the release of cytochrome c into cytoplasm and the generation of cleaved Caspase- 3 by

Western blot. As illustrated by [Fig. 6], after FAC (25-200 $\mu \mathrm{M})$ treatment for $120 \mathrm{~h}$, a dose-

dependent up-regulation of cytochrome $\mathrm{c}$ in this study was detected, which was accompanied by the generation of activated fragments of Caspase-3.

To investigate whether iron induces osteoblast apoptosis through alterations in Bcl-2 and Bax expression, Western blot were utilized to analyze Bax and Bcl-2 protein levels. In our present experiment, a dose-dependent down-regulation of Bcl-2, as well as an up-regulation of Bax was observed in osteoblasts exposed to FAC (25-200 $\mu \mathrm{M})$ for $120 \mathrm{~h}$ [Fig. 6].

\section{Depolarisation of MMP in osteoblasts due to iron}

Apoptosis could be initiated by the reduction of MMP, therefore it was decided to investigate whether such a reduction of MMP and consequent apoptosis is induced by iron. JC-1 dying of the osteoblasts and a flow cytometry were utilised to assess the amount of MMP. [Fig.7] shows how MMP was diminished with the application of FAC in various quantities for $120 \mathrm{~h}$. An increase in the strength of fluorescence of green JC-1 monomers compared to red JC-1 aggregates was considered to signify a significant reduction in MMP. [Fig. 7(C)] illustrates how the control group's MMP had no reduction following JC-1 dying, due to apparent diminished strength of green fluorescence compared to red fluorescence. However, a breakdown of MMP was seen to be associated with apoptotic osteoblasts caused by increased iron levels. An increased number of mitochondria were observed with a greater amount of green fluorescence, 
286

287

288

289

290

291

292

293

294

295

296

297

298

299

300

301

302

303

304

following the addition of FAC at varying concentrations of $25-200 \mu \mathrm{M}$, for a duration of $120 \mathrm{~h}$.

\section{$\mathrm{N}$-acetyl-L-cysteine (NAC)'s protection impact on iron-related apoptosis in osteoblasts}

This was to ascertain the extent to which NAC can prevent apoptotic occurrences related to iron in osteoblasts, by reducing the creation of ROS. The results suggested that the application of NAC was able to reduce the mitochondria's loss of cytochrome c, reduce the creation of ROS as an effect of iron to a large degree and consequently diminish the breakdown of MMP related to iron [Fig. 8]. As illustrated by [Fig. 8(E)], Caspase-3's stimulation was thus inhibited by NAC, Bcl-2/Bax modulation was inverted, while apoptotic osteoblasts resulting from the effects of iron were effectively reduced by NAC's application to osteoblasts. Therefore, apoptosis as a result of the effects of iron can also be seen to be significantly exacerbated by ROS production.

\section{Effects of iron on bone marrow-derived MSCs viability and apoptosis}

MSCs, characterised by their multipotent differentiation capacity, are recruited to the bone remodeling surface and differentiated into osteoblasts, which play an essential role to maintain bone mass. To test the effects of iron on the viability of MSCs in vitro, the CCK-8 assay kit was used in our study. As shown in [Fig. 9], iron inhibited the viability of MSCs at $72 \mathrm{~h}$ and $144 \mathrm{~h}$ in a dose-dependent manner. Next, we detected whether apoptosis was involved in iron-induced cellular toxicity. As reported in [Fig. 10], a significant increase of the apoptosis rates was observed in bone marrow-derived MSCs exposed with FAC $(200 \mu \mathrm{M})$ for $144 \mathrm{~h}$. Taken together, iron overload- induced toxicity of bone marrow-derived MSCs appears to be partially 
305 mediated by apoptosis.

306 Inhibitory effects of iron on osteogenic differentiation and mineralization

307 To investigate whether excess iron impaire osteogenesis of bone marrow-derived MSCs and

308 mineralization, cells were cultured in osteogenesis differentiation media alone or in the presence

309 of FAC (25- $200 \mu \mathrm{M})$ for 14 days. Next, the activity of ALP, a specific marker of osteogenic

310 differentiation, was detected in bone marrow-derived MSCs. [Fig. 11(A)] shows that iron caused

311 a concentration-dependent inhibitory effect of the activity of ALP in osteogenesis of MSCs. In

312 addtion, Alizarin Red staining showed that iron leaded to a concentration-dependent impairment

313 of mineralization. At the concentration of $200 \mu \mathrm{M}$, iron almost completely inhibited the

314 mineralization process of MSCs in vitro. Then, we estimated the effect of iron-overload on

315 calcium deposotion of the extracellular matrix. As demonstrated in [Fig. 11(B-D)], FAC caused a

316 decrease in the calcium content of the extracellular matrix in a dose-dependent manner,

317 which is accordance with the Alizarin Red staining results.

\section{Discussion}

321 due to conditions such as hemochromatosis and the thalassemia (Wong $P$ et al., 2013; Haidar $R$

322 et al., 2011; Valenti L et al., 2009). Meanwhile, various studies confirmed that age-associated

323 iron accumulation is a contributing factor in the pathogenesis of postmenopausal osteoporosis 
324 (Li GF et al., 2012; Fiona Mitchell et al., 2012). It has also been demonstrated that increased

325 physiological iron stores could accelerate bone loss, even in healthy adults (Kim BJ et al., 2012;

326 Fiona Mitchell et al., 2012). The maintenance of normal skeletal homeostasis relies on

327 osteoblasts, which is responsible for bone matrix synthesis, secretion and mineralization. The impairment of osteoblasts is considered to be a major factor contributing to osteoporosis in iron overload related diseases (Domrongkitchaiporn S et al., 2003; Terpos E et al., 2010;

Mahachoklertwattana $P$ et al., 2003; Doyard M et al., 2016). Therefore, it is essential to

investigate the toxic effects of iron on osteoblasts and elucidate the molecular mechanisms of

iron toxicity in these cells. Our present experiment reveals that iron-overload causes deterimental

effects in the osteoblastic cellular proliferation. We have also found that iron-overload

effectively induces apoptosis in osteoblasts in vitro, which is in accordance with the findings of

previously published research (Messer JG et al., 2009; Doyard M et al., 2012). More

interestingly, our study, for the first time, suggests that the apoptosis caused by iron overload is correlated with activation of the mitochondrial pathway.

While iron is a fundamental element for various crucial biological processes, such as enzymatic reactions and oxygen transport, excess iron accumulation could damge cells by catalyzing the over-production of damaging hydroxy radical through Haber-Weiss reactions (Dixon SJ et al., 2014). Labile iron pool, known as free and chelatable iron, is the major 342 potentially toxic form in iron-overload related diseases (Esposito BP et al., 2003; Devos D et al., 343 2014; Berdoukas Vet al., 2015; Chai X et al., 2015). In this study, we observed that the 
344 intracellular LIP level in osteoblasts was significantly increased after exposure to FAC for $120 \mathrm{~h}$.

345 ROS, which induced by labile iron via iron-dependent oxidase (for example, xanthine oxidase,

346 nicotinamide adenine dinucleotide phosphate hydride oxidases, lipoxygenases) or the Haber-

347 Weiss reaction, is primary responsible for cellular damage caused by iron overload (Dixon SJ et

348 al., 2014). Numerous studies reported that celluar and genomic oxidative damage were highly

349 correlated with elevated levels of labile iron in thalassemia patients (Brissot P et al., 2012;

350 Berdoukas V et al., 2015). Next, we evaluated the generation of ROS in osteoblasts after

exposure to FAC and found that iron-overload induced ROS production drastically increased.

Moreover, the over-production ROS was paralleled with the increase of intracellular labile iron and the cytotoxicity of osteoblasts. The generation of ROS is tightly regulated through various ways, including NADPH oxidases, phagocyte oxidase and mitochondrial electron transport chain (Sahoo S et al., 2016; Dikalov S et al., 2011; Zhou J et al., 2013). Emerging evidence indicates that NADPH oxidases are the primary generator of ROS in the skeletal system and Nox-derived ROS are key players which mediates osteoblasts dysfunction with osteoporosis (Manolagas SC et al., 2010; Schröder K et al., 2015). In our study, we found that iron overload could upregulate the expression of NADPH oxidase 4 (Nox4) in osteoblasts after treatment with FAC. In addition, heme (an iron derivative), as electron transporter, plays an important role for superoxide generation of NOX family NADPH oxidases (Bedard K et al., 2007). of ROS in osteoblasts. 
364

365

366

367

368

369

370

371

372

373

374

375

376

377

378

379

380

381

382

383

Previous studies have also shown that iron overload exerts deterimental effects on various

cell types and its mechanisms by which iron toxicity occurs are closely associated with

apoptosis(Chan S et al., 2013; Park J et al., 2015; Dussiot M et al., 2014). In this experiment, we demonstrated that iron-overload effectively induced apoptosis in osteoblasts. Furthermore, the activation of Caspase-3 was also observed after treatment with FAC. Although it is well established that iron-overload could induce apoptosis, its exact pathway in osteoblasts is still largely unknown. In iron-overload conditions, excess labile iron enters the mitochondria via the calcium uniporter, and then interacts with reactive oxygen intermediates leaked from mitochondrial respiratory chain through Fenton reactions, catalyzing powerful ROS to damage mitochondria (Chen MP et al., 2014; Sripetchwandee J et al., 2014; Pelizzoni I et al., 2011;

Uchiyama A et al., 2008). ROS catalyzed by labile iron elicits a range of detrimental effects in mitochondria, as following (1) impairment of mitochondrial respiratory enzyme activity; (2) decrease in ATP production; (3) loss of MMP; and (4) damage to mitochondrial DNA (mtDNA) (Ward RJ et al., 2014; Mallikarjun V et al., 2014; Al-Qenaei A et al., 2014; Santambrogio P et al., 2015; Rouault TA., 2016; Rines AK, Ardehali H. 2013). In this experiment, the inhibition of mitochondrial dehydrogenase activity was observed in osteoblasts exposed to FAC. Considering that ROS enhanced by iron overload could impair mitochondrial ultrastructure and disrupt its function, which might subsequently activate Caspase-3 through various molecular cascade reactions, we hypothesized that iron-overload induced apoptosis of osteoblasts might occur through the mitochondrial pathway. 
The mitochondrial apoptosis pathway has been well documented by many previous studies

385

386

387

388

389

390

391

392

393

394

395

396

397

398

399

400

401

402

403

404

(Czabotar PE et al., 2014). In the mitochondrial pathway, mitochondrial membrane

permeabilization, characterized by the loss of MMP, is considered as the most critical event

activating caspase and causes apoptosis (Fuchs Y et al., 2011; Kroemer G et al., 2007). After

mitochondrial membrane permeabilization induced by various apoptotic stimuli, Cyto c releases

from mitochondrial intermembrane space, subsequently binds APDF1 in cytosol, and then forms

apoptosome through recruiting and activating caspase 9. Eventually, caspase-9 cleaves and

activates caspase-3, resulting in the activation of apoptotic cell death (Fuchs Y et al., 2011;

Kroemer $G$ et al., 2007). Therefore, the changes of MMP in osteoblasts after treatment with FAC

were studied in details. Confocal microscopy observation indicated that iron overload led to a

dose-dependent decrease of MMP in osteoblasts. Furthermore, the depolarization of MMP was

subsequent accompanied with Cyto $\mathrm{C}$ release from mitochondria into the cytoplasm. Based on

above data, we then further to study the expression changes of other essential molecules

regulating the mitochondrial apoptosis pathway to prove our hypothesis. Bcl-2 family proteins

have been confirmed to control cellular apoptosis by directly or indirectly regulating

mitochondrial membrane permeabilization (Czabotar PE et al., 2014). Bcl-2, an anti-apoptotic

molecule, and Bax, an pro-apoptotic molecule, are key members among the Bcl-2 family

(Hardwick JM et al., 2013). The decrease in Bcl-2 or increase in Bax, could promote

permeabilization of the mitochondrial membrane, leading to the release of Cyto $\mathrm{c}$ and eventually

triggering apoptosis(Kroemer $G$ et al., 2007). In our study, we observed that iron overload

caused the upregulation of Bax and cleaved caspase-3, as well as the downregulation of Bcl-2.

PeerJ reviewing PDF | (2016:05:11057:1:0:REVIEW 6 Sep 2016) 
405 The changes of Bcl-2 and Bax expression in osteoblasts may have been sufficent to facilitate 406 mitchondrial membrane permeability. Taken together, our findings indicate that the 407 mitochondrial apoptosis pathway might be invovled, at least in part, in iron overload-releated 408 osteoblast injury.

NAC is a well-known antioxidant, which elevates the intracellular glutathione levels, an important module in the cellular antioxidative system ( Tsay J et al., 2010). In our study, we

411 found that apoptosis induced by iron overload in osteoblasts was associated with increased

412 harmful free radicals and was also largely prevented by NAC. Meanwhile, previous studies also 413 found that NAC could enhance osteogenesis and inhibit osteoclast differentiation (Yamada M et 414 al., 2013; Jun JH et al., 2008; Hyeon S et al., 2013; Lee NK et al., 2005). This suggests that 415 NAC could be an adjunctive therapy in iron-overload related bone loss. The concept that 416 increased harmful free radicals induced by iron overload is the main contributer of iron toxicity 417 is not new. But, to our knowledge, detailed mechanism for NAC protection against iron 418 overload-induced osteoblasts apoptosis has not been reported. Our findings revealed that NAC 419 could prevent the mitochondria damage casused by iron overload through directly scavenge the over-generated ROS. Thus, cytochrome c released from mitochondria was decrease and the the activation of caspase- 3 was inhibited. In addition, after NAC treatment, the expression of Bcl-2 was markedly increase, while the expression of Bax was decrease. This result might imply that 
425 studies were conducted in vitro; as such our results may not be representative of biological

426 process in vivo. Furthermore, as human osteoblastic cells are difficult to obtain, we utilized the

427 MC3T3-E1 osteoblastic cell line to examine the toxicity of iron in this study. Although

428 numerous studies have reported that the MC3T3-E1 cell line is similar to human osteoblasts in

429 function(Czekanska EM et al., 2012), further studies using human osteoblasts are warranted.

430 Finally, our results indicate that the mitochondrial apoptotic pathway is involved in mediating

431 iron toxicity in osteoblasts. However, the iron-mediated destabilization of lysosomal membranes

432 represents an alternative mechanism of iron toxicity. In future experiments, we aim to explore

433 the potential effects of iron on lysosomes, which may include, lysosomal membrane

434 permeabilization and cross-talk between lysosomes and mitochondria.

In the maintenance of skeletal homeostasis, besides osteoblast, mesenchymal stem cell also also plays an essential role in osteogensis. It has been reported that both the number and osteogenic differentiation potential of bone marrow-derived MSCs decrease in osteoporotic patients(Xian L et al., 2012; Guan M et al., 2012). In our experiments, we found that iron caused 439 a concentration-dependent inhibitory effect of the viability of bone marrow-derived MSCs.

440 Furthermore, iron overload in bone marrow-derived MSCs result in increased apoptosis. This is 441 similar to our results in osteoblasts and also consistent with previous reports (Chai X et al., 2015;

442 Zhang Yet al., 2015; Lu W et al., 2013). To explore the effect of iron-overload on the osteogenic 443 differentiation of bone marrow-derived MSCs, we estimated the change of ALP activity. In 444 response to osteogenic induction, bone marrow-derived MSCs could increase the activity of ALP, 
445 a specific marker of osteogenic differentiation. In iron-overload condition, this response was

446 significantly attenuated. In addition, iron could directly inhibit matrix mineralization of bone

447 marrow-derived MSCs. Numerous clinical and in vivo studies have also indicated that defective

448 mineralization of bone was one of the pathological changes in iron overload-related

449 osteoporosis(Doyard M et al., 2016; Mahachoklertwattana P et al., 2003; Matsushima S et al.,

450 2003). Our in vitro findings that excess iron caused MSCs apoptosis and impaired osteogenic

451 differentiation and mineralization might, at least in part, offer understanding of low bone density

452 in iron overload diseases.

453 Overall, our data indicate that iron significantly induces apoptosis in osteoblasts in vitro.

454 NAC could remarkably relieve iron overload-induced osteoblasts apoptosis. In addition, we 455 demonstrate that iron induces apoptosis via the enhanced production of ROS, which impairs 456 mitochondrial function and leads to MMP collapse, cytochrome c release, and caspase activation.

457 This provides valuable insights into the molecular mechanisms underlying osteoblastic cell death 458 in the iron-overload condition. Meanwhile, we also revealed that iron overload could promote 459 apoptosis and impair osteogenic differentiation and mineralization in bone marrow-derived 460 MSCs. 


\section{Acknowledgments}

462 None.

\section{References}

464

465

466

467

468

469

470

471

472

473

474

475

476

477

478

479

- Vogiatzi MG, Macklin EA, Fung EB, Vichinsky E, Olivieri N, Kwiatkowski J, Cohen A, Neufeld E, Giardina PJ. 2006. Prevalence of fractures among the Thalassemia syndromes in North America. Bone. Apr;38(4):571-5. Epub 2005 Nov 17.

- Vogiatzi MG, Macklin EA, Fung EB, Cheung AM, Vichinsky E, Olivieri N, Kirby M, Kwiatkowski JL, Cunningham M, Holm IA, Lane J, Schneider R, Fleisher M,Grady RW, 9 Peterson CC, Giardina PJ; Thalassemia Clinical Research Network. 2009 Bone disease in thalassemia: a frequent and still unresolved problem. J Bone Miner Res. Mar;24(3):543-57. 1 doi: $10.1359 / \mathrm{jbmr} .080505$. men:3-yearretrospective longitudinal study. J Bone Miner Res. Nov;27(11):2279-90. doi: $10.1002 / \mathrm{jbmr} .1692$.

- Wong P, Fuller PJ, Gillespie MT, Kartsogiannis V, Kerr PG, Doery JC, Paul E, Bowden DK, Strauss BJ, Milat F. 2014 Thalassemia bone disease: a 19-year longitudinal analysis. J Bone Miner Res. Nov;29(11):2468-73. doi: 10.1002/jbmr.2266.

- Yang Q, Jian J, Abramson SB, Huang X. 2011 Inhibitory effects of iron on bone 

morphogenetic protein 2-induced osteoblastogenesis. J Bone Miner Res. Jun;26(6):1188-96. doi: $10.1002 /$ jbmr.337. Doty SB, Grady RW, Giardina PJ, Boskey AL, Vogiatzi MG. 2010. Bone loss caused by iron overload in a murine model: importance of oxidative stress.Blood. 7;116(14):2582-9. doi: 10.1182/blood-2009-12-260083. genes and proteins, down-regulates osteoblastic phenotype,and is associated with apoptosis in fetal rat calvaria cultures. Bone ;45(5):972-9. doi: 10.1016/j.bone.2009.07.073. doi: 10.1007/s00198-011-1871-z. Z, He G, Luo X. 2016. Activation of GLP-1 Receptor Promotes

Stem Cell Reports. Apr 12;6(4):633. doi: 10.1016/j.stemcr.2016.03.010. decreases osteoblastic activity in human MG-63 cells. Osteoporos Int. Oct;23(10):2435-45.

- Meng J, Ma X, Wang N, Jia M, Bi L, Wang Y, Li M, Zhang H, Xue X, Hou Z, Zhou Y, Yu

Bone Marrow Stromal Cell Osteogenic Differentiation through $\beta$-Catenin. 
Nov 11;147(4):742-58. doi: 10.1016/j.cell.2011.10.033.

499

500

501

502

503

504

505

506

507

508

509

510

511

512

513

514

515

- Green DR, Galluzzi L, Kroemer G. Cell biology. Metabolic control of cell death. 2014.

Science. Sep 19;345(6203):1250256. doi: 10.1126/science.1250256.

- Tait SW, Green DR. 2013. Mitochondrial regulation of cell death. Cold Spring Harb

Perspect Biol. Sep 1;5(9). pii: a008706. doi: 10.1101/cshperspect.a008706.

- Lill R. 2009. Function and biogenesis of iron-sulphur proteins. Nature. Aug 13;460(7257):831-8. doi: 10.1038/nature08301.

- Dixon SJ, Stockwell BR. 2014. The role of iron and reactive oxygen species in cell death. Nat Chem Biol. Jan;10(1):9-17. doi: 10.1038/nchembio.1416.

- Pietrangelo A. 2016. Mechanisms of iron hepatotoxicity. J Hepatol. Feb 5. pii: S01688278(16)00080-5. doi: 10.1016/j.jhep.2016.01.037.

- Zarjou A, Jeney V, Arosio P, Poli M, Zavaczki E, Balla G, Balla J. 2010. Ferritin ferroxidase activity: a potent inhibitor of osteogenesis. J Bone Miner Res. Jan;25(1):164-72. doi: 10.1359/jbmr.091002.

- Ding F, Shao ZW, Yang SH, Wu Q, Gao F, Xiong LM. 2012. Role of mitochondrial pathway in compression-induced apoptosis of nucleus pulposus cells. 
$516 \quad$ Apoptosis; 17:579e90.18.

517

518

519

520

521

522

523

524

525

526

527

528

529

530

531

532

- Cai XY, Xia Y, Yang SH, Liu XZ, Shao ZW, Liu YL, Yang W, Xiong LM. 2015.

Ropivacaine- and bupivacaine-induced death of rabbit annulus fibrosus cells in vitro: involvement of the mitochondrial apoptotic pathway. Osteoarthritis Cartilage. Oct;23(10):1763-75. doi: 10.1016/j.joca.2015.05.013.

- Henry CM, Hollville E, Martin SJ. 2013. Measuring apoptosis by microscopy and flow cytometry. Methods. Jun 1;61(2):90-7. doi: 10.1016/j.ymeth.2013.01.008.

- Zhu Y, Mao Z, Gao C. 2013. Control over the gradient differentiation of rat BMSCs on a PCL membrane with surface-immobilized alendronate gradient.

Biomacromolecules. Feb 11;14(2):342-9. doi: 10.1021/bm301523p.

- Zhang X, Li J, Nie J, Jiang K, Zhen Z, Wang J, Shen L. 2010. Differentiation character of adult mesenchymal stem cells and transfection of MSCs

withlentiviral vectors. J Huazhong Univ Sci Technolog Med Sci. Dec;30(6):687-93. doi: 10.1007/s11596-010-0641-Z.

- Malladi P, Xu Y, Chiou M, Giaccia AJ, Longaker MT. 2006. Effect of reduced oxygen tension on chondrogenesis and osteogenesis in adipose-derived mesenchymal cells. Am J Physiol Cell Physiol. Apr;290(4):C1139-46. 
533

534

535

536

537

538

539

540

541

542

543

544

545

546

547

548

549

550

551

- Lyu Z, Wang H, Wang Y, Ding K, Liu H, Yuan L, Shi X, Wang M, Wang Y, Chen H. 2014. Maintaining the pluripotency of mouse embryonic stem cells on gold nanoparticle layers with nanoscale but not microscale surface roughness. Nanoscale. Jun 21;6(12):6959-69. doi: 10.1039/c4nr01540a.

- Tenopoulou M, Kurz T, Doulias PT, Galaris D, Brunk UT. 2007. Does the calcein-AM method assay the total cellular 'labile iron pool' or only a fraction of it? Biochem J. Apr $15 ; 403(2): 261-6$.

- Glickstein H, El RB, Shvartsman M, Cabantchik ZI. 2005. Intracellular labile iron pools as direct targets of iron chelators: fluorescence study of chelator action in livingcells. Blood. Nov 1;120(9):3242-50.

- Kaur D, Lee D, Ragapolan S, Andersen JK. 2009. Glutathione depletion in immortalized midbrain-derived dopaminergic neurons results in increases in the labile iron pool: implications for Parkinson's disease. Free Radic Biol Med. Mar 1;46(5):593-8. doi: 10.1016/j.freeradbiomed.2008.11.012.

- Brissot P, Ropert M, Le Lan C, Loréal O. 2012. Non-transferrin bound iron: a key role in iron overload and iron toxicity. Biochim Biophys Acta. Mar;1820(3):403-10. doi: 10.1016/j.bbagen.2011.07.014.

- Wong P, Fuller PJ, Gillespie MT, Kartsogiannis V, Strauss BJ, Bowden D, Milat F. 2013. Thalassemia bone disease: the association between nephrolithiasis, bone mineral density and 

patients with $\beta$ thalassemia major. Bone. Mar 1;48(3):425-32. doi: 10.1016/j.bone.2010.10.173. Osteoporos Int. Apr;20(4):549-55. doi: 10.1007/s00198-008-0701-4. implications. Osteoporos Int. Oct;23(10):2403-8. doi: 10.1007/s00198-012-1982-1. Endocrinology 8, 506 (September) | doi:10.1038/nrendo.2012.127

- Domrongkitchaiporn S, Sirikulchayanonta V, Angchaisuksiri P, Stitchantrakul W, Kanokkantapong C, Rajatanavin R. 2003. Abnormalities in bone mineral density and bone histology in thalassemia. J Bone Miner Res. Sep;18(9):1682-8.

- Terpos E, Voskaridou E. 2010. Treatment options for thalassemia patients with osteoporosis. Ann N Y Acad Sci. Aug;1202:237-43. 
570 histomorphometry in children and adolescents with beta-thalassemia disease: iron-associated 571 focal osteomalacia. J Clin Endocrinol Metab. Aug;88(8):3966-72.

572

573

574

575

576

577

578

579

580

581

582

583

584

585

586

587

588

- Esposito BP, Breuer W, Sirankapracha P, Pootrakul P, Hershko C, Cabantchik ZI. 2003. Labile plasma iron in iron overload: redox activity and susceptibility to chelation. Blood. Oct 1;102(7):2670-7.

- Devos D, Moreau C, Devedjian JC, Kluza J, Petrault M, Laloux C, Jonneaux A, Ryckewaert G, Garçon G, Rouaix N, Duhamel A, Jissendi P, Dujardin K, Auger F, Ravasi L, Hopes L, Grolez G, Firdaus W, Sablonnière B, Strubi-Vuillaume I, Zahr N, Destée A, Corvol JC, Pöltl D, Leist M, Rose C, Defebvre L, Marchetti P,Cabantchik ZI, Bordet R. 2014. Targeting chelatable iron as a therapeutic modality in Parkinson's disease. Antioxid Redox Signal. Jul 10;21(2):195-210. doi: 10.1089/ars.2013.5593.

- Berdoukas V, Coates TD, Cabantchik ZI. 2015. Iron and oxidative stress in cardiomyopathy in thalassemia. Free Radic Biol Med. Nov;88(Pt A):3-9. doi:

10.1016/j.freeradbiomed.2015.07.019.

- Chai X, Li D, Cao X, Zhang Y, Mu J, Lu W, Xiao X, Li C, Meng J, Chen J, Li Q, Wang J, Meng A, Zhao M. 2015. ROS-mediated iron overload injures the hematopoiesis of bone 

doi: $10.1038 /$ srep10181.

591

592

593

594

595

596

597

598

599

600

601

602

603

604

605

606

- Sahoo S, Meijles DN, Pagano PJ. 2016. NADPH oxidases: key modulators in aging and agerelated cardiovascular diseases? Clin Sci (Lond). Mar 1;130(5):317-35. doi: $10.1042 / \mathrm{CS} 20150087$.

- Dikalov S. 2011. Cross talk between mitochondria and NADPH oxidases. Free Radic Biol Med.1;51(7):1289-301. doi: 10.1016/j.freeradbiomed.2011.06.033.

- Zhou J, Ye S, Fujiwara T, Manolagas SC, Zhao H. 2013. Steap4 plays a critical role in osteoclastogenesis in vitro by regulating cellular iron/reactive oxygen species (ROS) levels and cAMP response element-binding protein (CREB) activation. J Biol Chem. Oct 18;288(42):30064-74. doi: 10.1074/jbc.M113.478750.

- Manolagas SC. 2010. From estrogen-centric to aging and oxidative stress: a revised perspective of the pathogenesis of osteoporosis. Endocr Rev. Jun;31(3):266-300. doi: 10.1210/er.2009-0024.

- Schröder K. 2015. NADPH oxidases in bone homeostasis and osteoporosis. Cell Mol Life Sci. Jan;72(1):25-38. doi: 10.1007/s00018-014-1712-2.

- Bedard K, Krause KH. 2007. The NOX family of ROS-generating NADPH oxidases: physiology and pathophysiology. Physiol Rev. Jan;87(1):245-313. 
607

608

- Chan S, yu Ye J, Chan G C F. 2013. TPO exerts a protective effect on iron-overload induces apoptosis in cardiomyocytes via mitochondrial pathways. Blood, 122(21): 4668-4668.

- Park J, Lee DG, Kim B, Park SJ, Kim JH, Lee SR, Chang KT, Lee HS, Lee DS. 2015. Iron overload triggers mitochondrial fragmentation via calcineurin-sensitive signals in HT-22 hippocampal neuron cells. Toxicology. Nov 4;337:39-46. doi: 10.1016/j.tox.2015.08.009.

- Dussiot M, Maciel TT, Fricot A, Chartier C, Negre O, Veiga J, Grapton D, Paubelle E, Payen E, Beuzard Y, Leboulch P, Ribeil JA, Arlet JB, Coté F, Courtois G, Ginzburg YZ, Daniel TO, Chopra R, Sung V, Hermine O, Moura IC. 2014. An activin receptor IIA ligand trap corrects ineffective erythropoiesis in $\beta$-thalassemia. Nat Med. Apr;20(4):398-407. doi: 10.1038/nm.3468.

- Chen MP, Cabantchik ZI, Chan S, Chan GC, Cheung YF. 2014 Iron overload and apoptosis of HL-1 cardiomyocytes: effects of calcium channel blockade. PLoS One. 12;9(11):e112915. doi: 10.1371/journal.pone.0112915. eCollection 2014.

- Sripetchwandee J, KenKnight SB, Sanit J, Chattipakorn S, Chattipakorn N. 2014. Blockade of mitochondrial calcium uniporter prevents cardiac mitochondrial dysfunction caused by iron overload. Acta Physiol (Oxf). Feb;210(2):330-41. doi: 10.1111/apha.12162.

- Pelizzoni I, Macco R, Morini MF, Zacchetti D, Grohovaz F, Codazzi F. 2011. Iron handling in hippocampal neurons: activity-dependent iron entry andmitochondria-mediated neurotoxicity. Aging Cell. Feb;10(1):172-83. doi: 10.1111/j.1474-9726.2010.00652.x. 
626

627

628

629

630

631

632

633

634

635

636

637

638

639

640

641

642

643

644

- Uchiyama A, Kim JS, Kon K, Jaeschke H, Ikejima K, Watanabe S, Lemasters JJ. 2008. Translocation of iron from lysosomes into mitochondria is a key event during oxidative stress-induced hepatocellular injury. Hepatology. Nov;48(5):1644-54. doi: 10.1002/hep. 22498 .

- Ward RJ, Zucca FA, Duyn JH, Crichton RR, Zecca L. 2014. The role of iron in brain ageing and

neurodegenerative disorders. Lancet Neurol. Oct;13(10):1045-60.

doi:10.1016/S1474-4422(14)70117-6.

- Mallikarjun V, Sriram A, Scialo F, Sanz A. 2014. The interplay between mitochondrial protein and iron homeostasis and its possible role in ageing. Exp Gerontol. Aug;56:123-34. doi: 10.1016/j.exger.2013.12.015.

- Al-Qenaei A, Yiakouvaki A, Reelfs O, Santambrogio P, Levi S, Hall ND, Tyrrell RM, Pourzand C. 2014. Role of intracellular labile iron, ferritin, and antioxidant defence in resistance of chronically adapted Jurkat T cells to hydrogen peroxide. Free Radic Biol Med. Mar;68:87-100. doi: 10.1016/j.freeradbiomed.2013.12.006.

- Santambrogio P, Dusi S, Guaraldo M, Rotundo LI, Broccoli V, Garavaglia B, Tiranti V, Levi S. 2015. Mitochondrial iron and energetic dysfunction distinguish fibroblasts and induced neurons from pantothenatekinase-associated neurodegeneration patients. Neurobiol Dis. Sep;81:144-53. doi: 10.1016/j.nbd.2015.02.030. 
645 646 Dev.Mar 25;38:31-37. doi: 10.1016/j.gde.2016.02.004.

647

648

649

650

651

652

653

654

655

656

657

658

659 Biochem. Mar 1;103(4):1246-55.

660

$662 \quad 10.1016 /$ j.freeradbiomed.2013.08.005. Mol Cell Cardiol. Feb;55:50-7. doi: 10.1016/j.yjmcc.2012.05.014. Jan;15(1):49-63. doi: 10.1038/nrm3722. 661 promotes RANKL-inducedosteoclast differentiation. Free Radic Biol Med. 65:789-99. doi:

- Rouault TA. 2016. Mitochondrial iron overload: causes and consequences. Curr Opin Genet

- Rines AK, Ardehali H. 2013. Transition metals and mitochondrial metabolism in the heart. J

- Czabotar PE, Lessene G, Strasser A, Adams JM. 2014. Control of apoptosis by the BCL-2 protein family: implications for physiology and therapy. Nat Rev Mol Cell Biol.

- Hardwick JM, Soane L. 2013. Multiple functions of BCL-2 family proteins. Cold Spring Harb Perspect Biol. Feb 1;5(2). pii: a008722. doi: 10.1101/cshperspect.a008722.

- Yamada M, Tsukimura N, Ikeda T, Sugita Y, Att W, Kojima N, Kubo K, Ueno T, Sakurai K, Ogawa T. 2013. N-acetyl cysteine as an osteogenesis-enhancing molecule for bone regeneration. Biomaterials. Aug;34(26):6147-56. doi: 10.1016/j.biomaterials.2013.04.064.

- Jun JH, Lee SH, Kwak HB, Lee ZH, Seo SB, Woo KM, Ryoo HM, Kim GS, Baek JH. 2008.

$58 \mathrm{~N}$-acetylcysteine stimulates osteoblastic differentiation of mouse calvarial cells. J Cell

- Hyeon S, Lee H, Yang Y, Jeong W. 2013. Nrf2 deficiency induces oxidative stress and 
663

664

665

666

667

668

669

670

671

672

673

674

675

676

677

678

679

680

681

- Lee NK, Choi YG, Baik JY, Han SY, Jeong DW, Bae YS, Kim N, Lee SY. 2005. A crucial role for reactive oxygen species in RANKL-induced osteoclastdifferentiation. Blood. Aug $1 ; 120(3): 852-9$

- Czekanska EM, Stoddart MJ, Richards RG, Hayes JS. 2012. In search of an osteoblast cell model for in vitro research. Eur Cell Mater. Jul 9;24:1-17.

- Ma KG, Shao ZW, Yang SH, Wang J, Wang BC, Xiong LM, Wu Q, Chen SF. Autophagy is activated in compression-induced cell degeneration and is mediated by reactive oxygen species innucleus pulposus cells exposed to compression. Osteoarthritis Cartilage. 2013 Dec;21(12):2030-8. doi: 10.1016/j.joca.2013.10.002.

- Xian L, Wu X, Pang L, Lou M, Rosen CJ, Qiu T, Crane J, Frassica F, Zhang L, Rodriguez JP, Xiaofeng Jia, Shoshana Yakar, Shouhong Xuan, Argiris Efstratiadis, Mei Wan, Xu Cao. 2012. Matrix IGF-1 maintains bone mass by activation of mTOR in mesenchymal stem cells. Nat Med. Jul;18(7):1095-101. doi: 10.1038/nm.2793.

- Guan M, Yao W, Liu R, Lam KS, Nolta J, Jia J, Panganiban B, Meng L, Zhou P, Shahnazari M, Ritchie RO, Lane NE. 2012. Directing mesenchymal stem cells to bone to augment bone formation and increase bone mass. Nat Med. Feb 5;18(3):456-62. doi: 10.1038/nm.2665.

- Zhang Y, Zhai W, Zhao M, Li D, Chai X, Cao X, Meng J, Chen J, Xiao X, Li Q, Mu J, Shen J, Meng A. 2015. Effects of iron overload on the bone marrow microenvironment in mice. PLoS One. Mar 16;10(3):e0120219. doi: 
683

684

685

686

687

688

689

690

691

692

693

694

695

696

697

698

699

- Mahachoklertwattana P, Sirikulchayanonta V, Chuansumrit A, Karnsombat P, Choubtum L, Sriphrapradang A, Domrongkitchaiporn S, Sirisriro R, Rajatanavin R. 2003. Bone histomorphometry in children and adolescents with beta-thalassemia disease: iron-associated focal osteomalacia. J Clin Endocrinol Metab. Aug;88(8):3966-72.

- Matsushima S, Torii M, Ozaki K, Narama I. 2003. Iron lactate-induced osteomalacia in association with osteoblast dynamics. Toxicol Pathol. Nov-Dec;31(6):646-54.

- Lu W, Zhao M, Rajbhandary S, Xie F, Chai X, Mu J, Meng J, Liu Y, Jiang Y, Xu X, Meng A. 2013. Free iron catalyzes oxidative damage to hematopoietic cells/mesenchymal stem cells in vitro and suppresses hematopoiesis in iron overload patients. Eur J Haematol. Sep;91(3):249-61. doi: 10.1111/ejh.12159. 


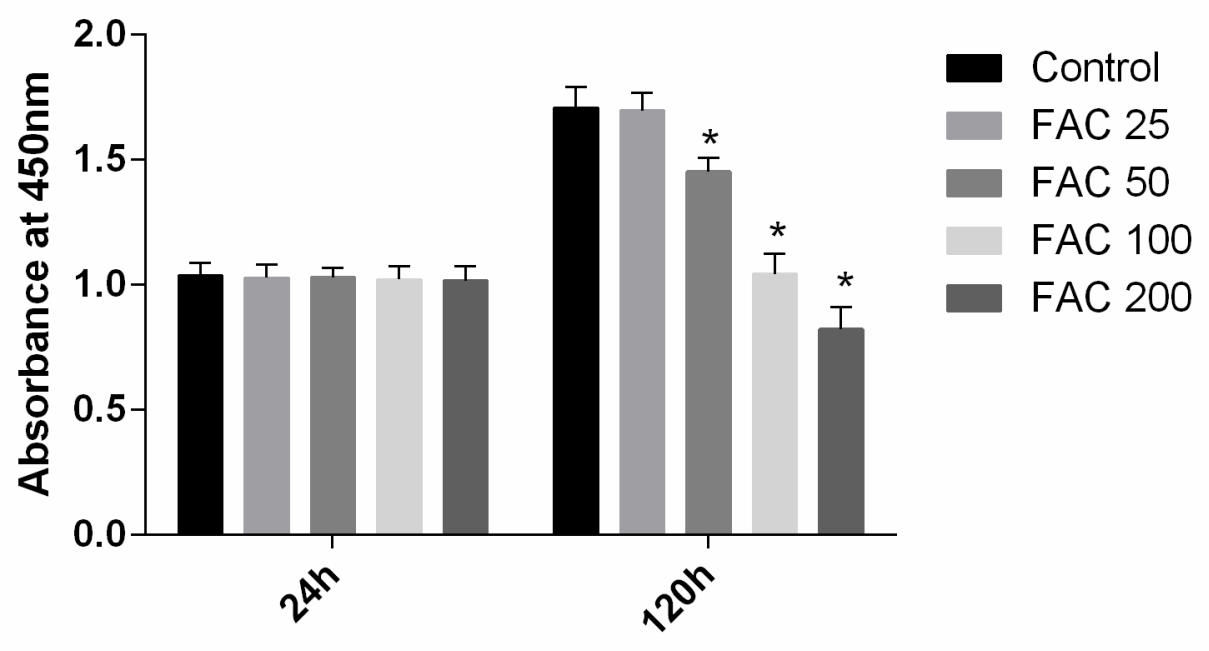

702 Figure 1 Cytotoxic effects of iron on the viability of osteoblasts. h FAC treatment. The values are presented as means $\pm \mathrm{SD}, \mathrm{n}=3 ; * \mathrm{P}<0.05$ vs. the control. 
A
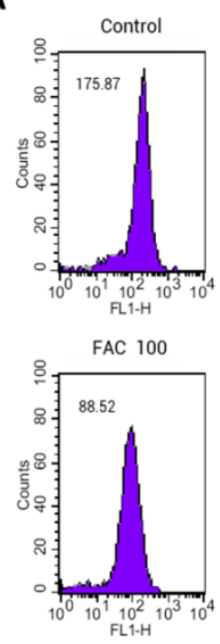

C
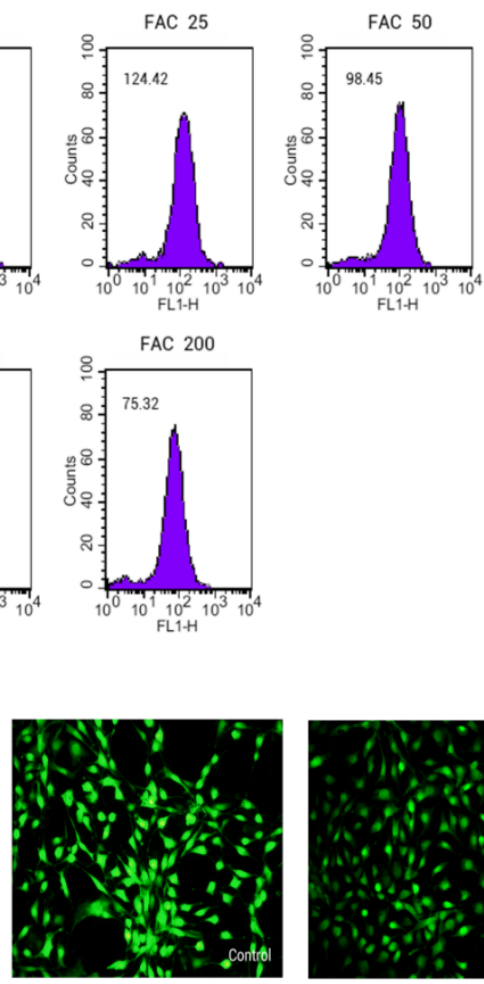

B
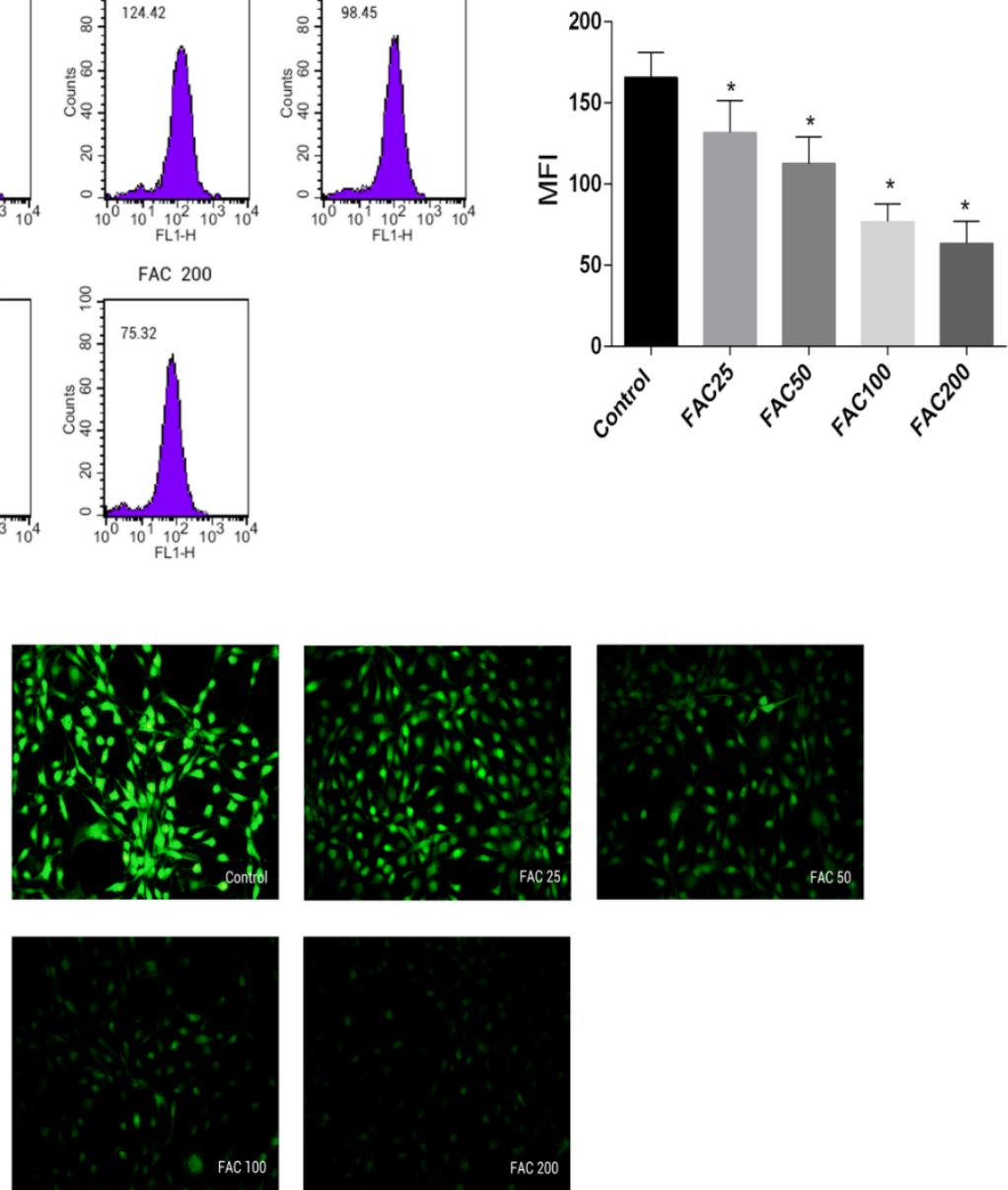

707

Figure 2 Effect of iron-overload on the intracellular LIP in osteoblasts.

709

710

711

712

713

714
The intracellular LIP in osteoblasts markedly increased after treatment with $0-200 \mu \mathrm{M} \mathrm{FAC}$ for $120 \mathrm{~h}$.

(A) Representative flow cytometric results for intracellular LIP after FAC treatment. The intracellular LIP was estimated by calcein-AM, a fluorescent iron-sensitive probe. The probe fluorescence was quenched after chelating with labile iron; the mean fluorescence intensity (MFI) measured by flow cytometry was negatively correlated with intracellular LIP. (B) The reduction in MFI indicated an elevation in the intracellular LIP in the osteoblasts. Data are presented as the means $\pm \mathrm{SD}, \mathrm{n}=3 ; * \mathrm{P}<$ 
715

716

717

718

0.05 vs. the control. (C) Representative fluorescence microscopy photomicrographs of intracellular LIP in osteoblasts. The quenching of green fluorescence indicates that the intracellular LIP was increased in cells.

A
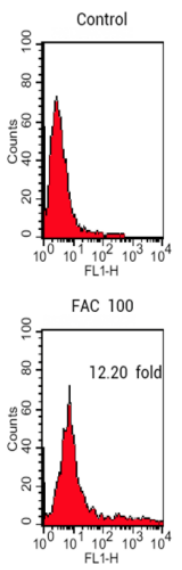
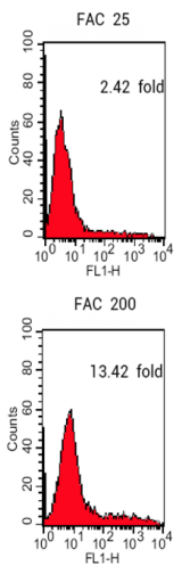

B

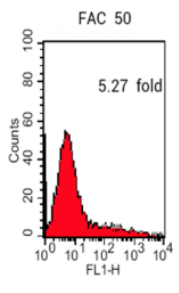

C

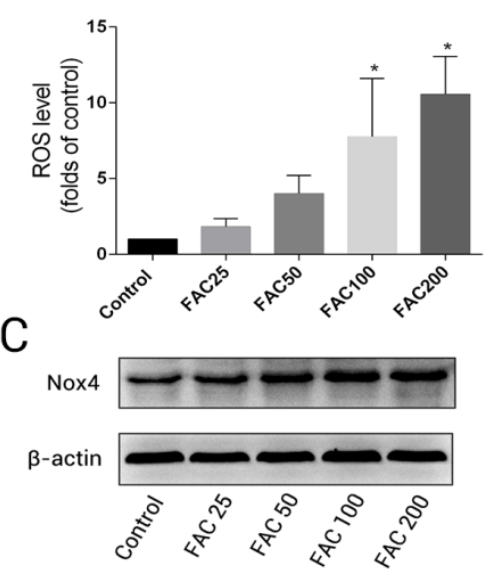

\section{Figure 3 Iron induced ROS generation and upregulation of Nox4 in osteoblasts.}

(A) Representative data of flow cytometric measurement of ROS production after labeling with H2DCF-DA. (B) Statistical bar graphs show the ROS levels in osteoblasts. The fluorescence intensity of osteoblasts was expressed relative to untreated osteoblasts (FAC $0 \mu \mathrm{M})$. Date are presented as means $\pm \mathrm{SD}, \mathrm{n}=3 ;{ }^{*} \mathrm{P}<0.05$ vs. the control. (C) Representative western blot data for Nox4 in osteoblasts following exposure to $\mathrm{FAC}(0-200 \mu \mathrm{M})$ for $120 \mathrm{~h}$. GAPDH was used as an internal control. 
A
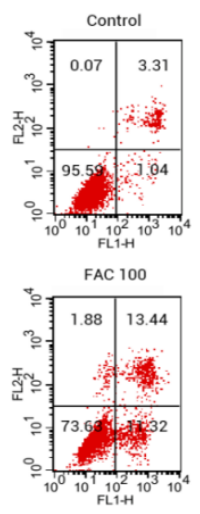

C
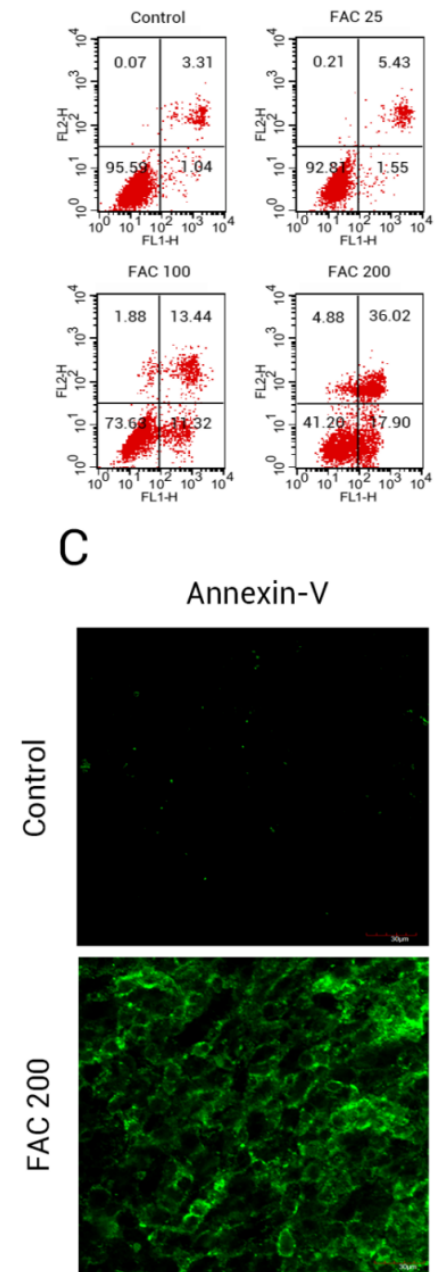

B
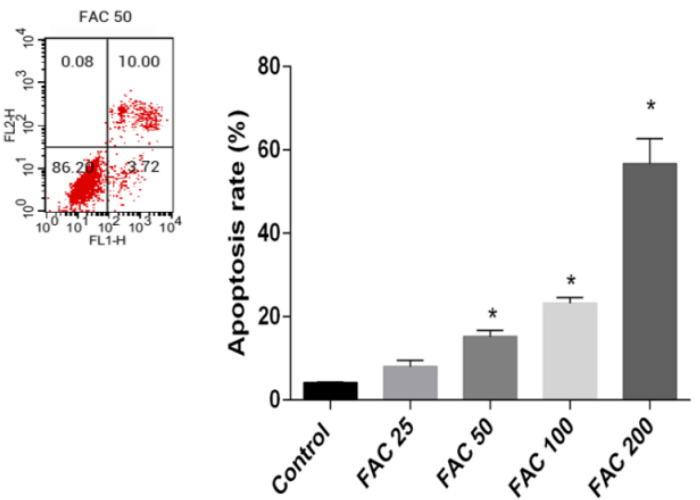

PI
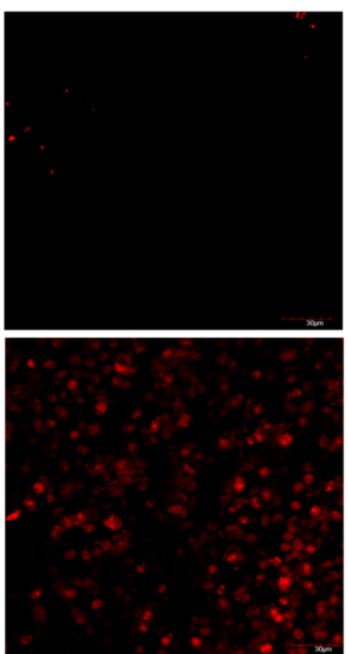

Merge
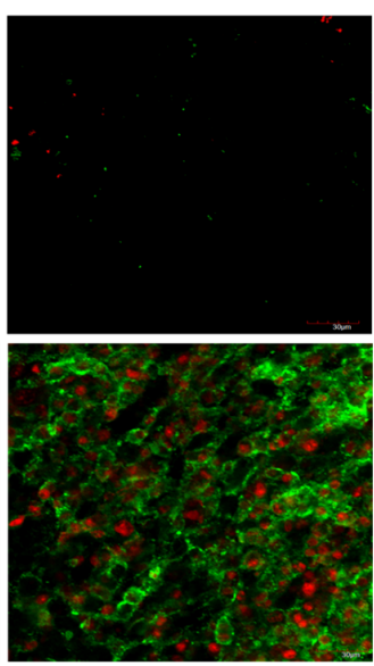

\section{Figure 4 Iron induced apoptosis in osteoblasts.}

727

(A) Representative flow cytometric analysis of apoptotic osteoblasts stained for Annexin V/PI after exposure to $0-200 \mu \mathrm{M}$ FAC for $120 \mathrm{~h}$. In each plot, the lower left quadrant represents live osteoblasts, the lower right and upper right quadrants represent apoptotic osteoblasts, and the upper left quadrant represents necrotic osteoblasts. (B) Statistical bar graphs show the mean values of flow cytometry data. Data are presented as the means $\pm \mathrm{SD}, \mathrm{n}=3 .{ }^{*} \mathrm{P}<0.05$ vs. the control. (C) Representative photomicrograph (OLYMPUS FV1000, Japan) of osteoblasts stained with AnnexinV/PI dye after 

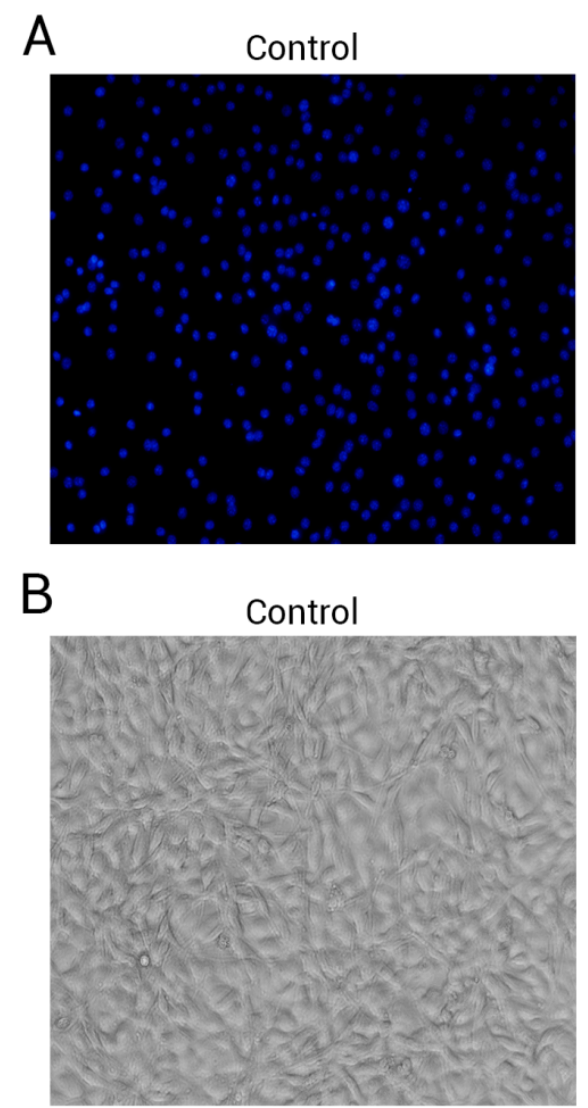

FAC 200

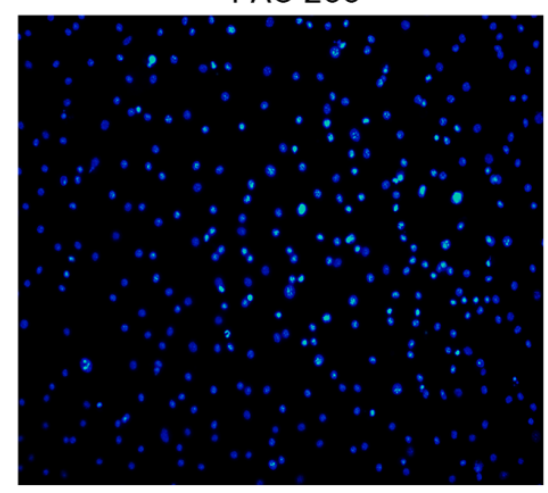

FAC 200

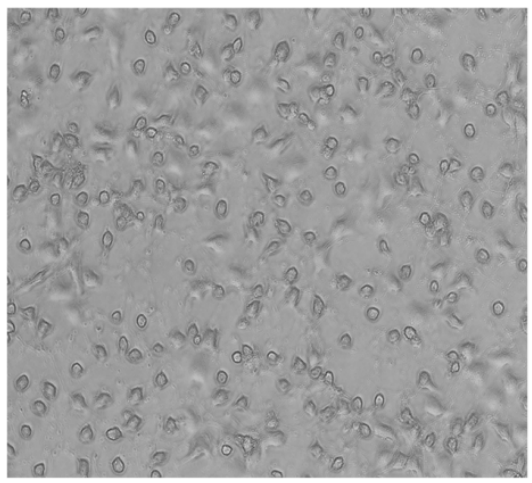




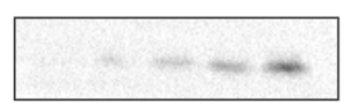

Cleaved Caspase-3

GAPDH

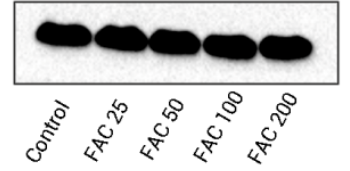

Cytochrome c

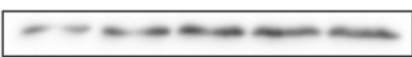

Bax

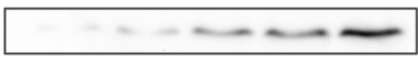

$\mathrm{Bcl}-2$

GAPDH

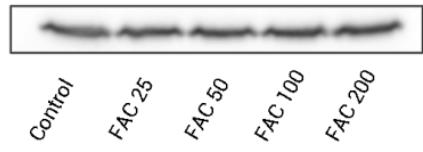

Figure 6 The expression of apoptosis-related proteins in osteoblasts. 
A
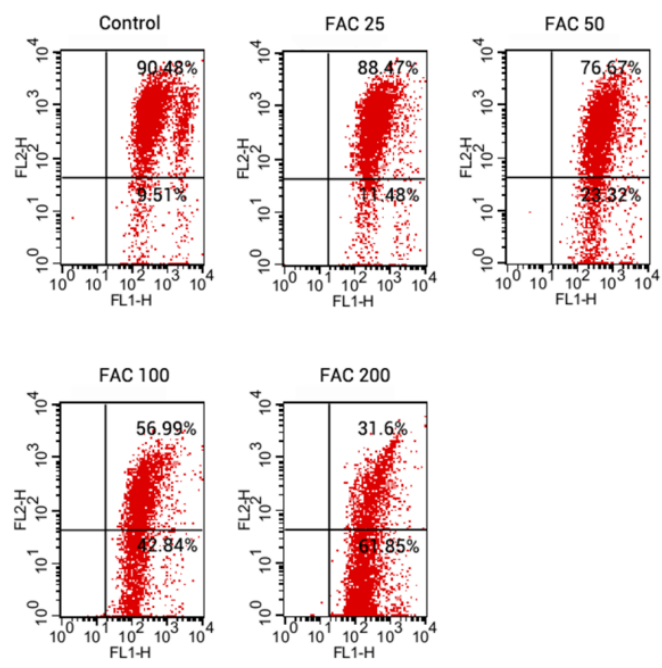

C
B
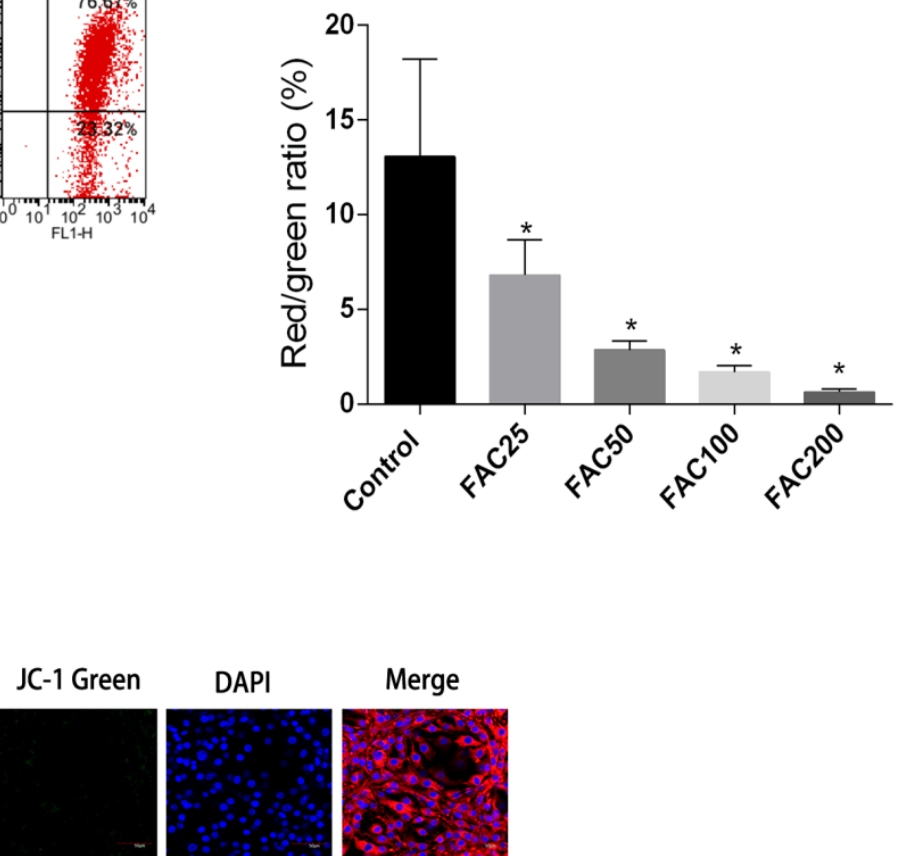

\section{Merge}
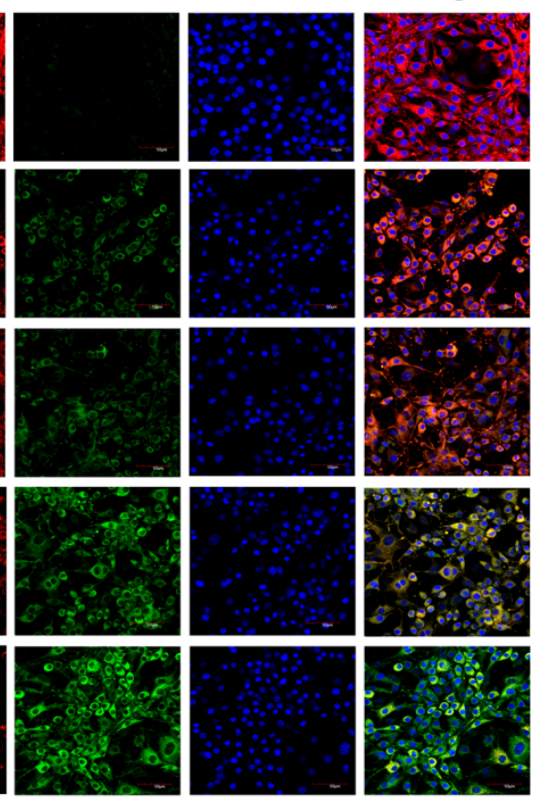

FAC 200

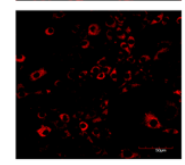

Figure 7 Iron-induced decrease in MMP of osteoblasts. 
A

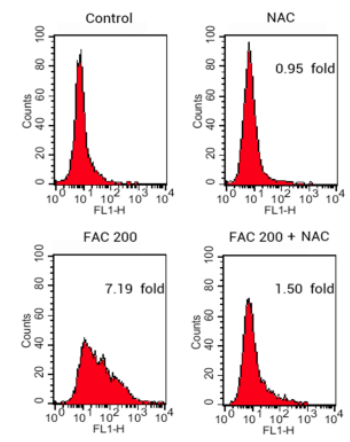

B
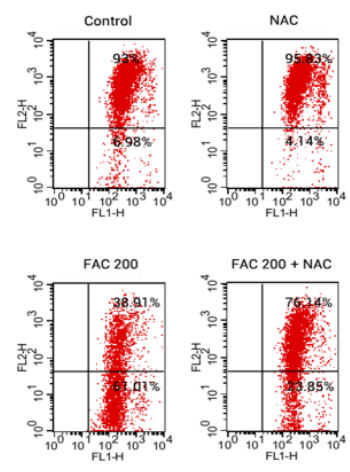

C
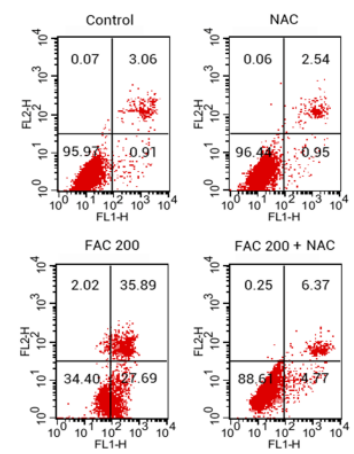
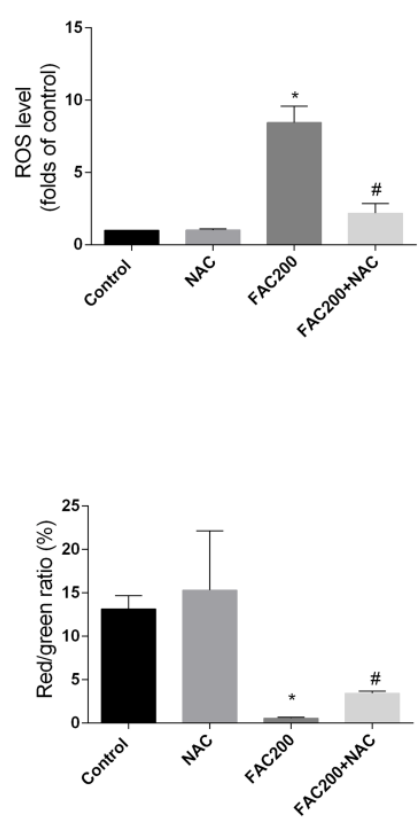

$E$
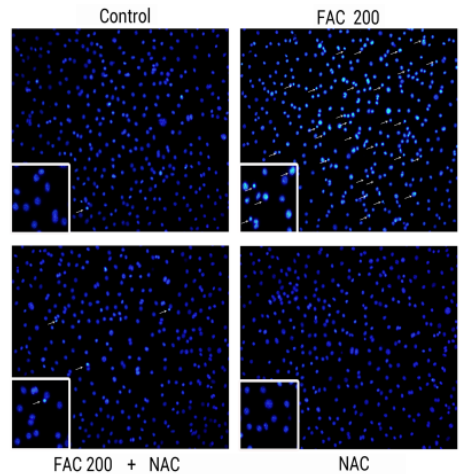

Control

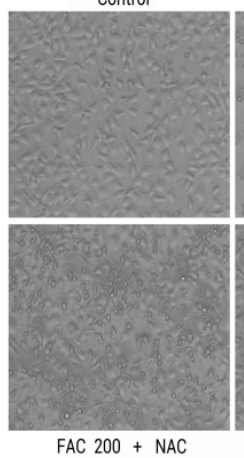

FAC 200

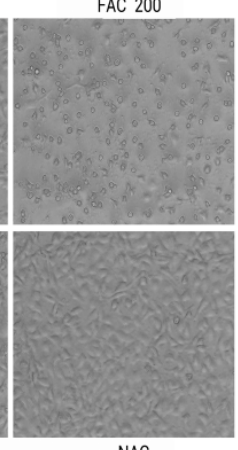

NAC

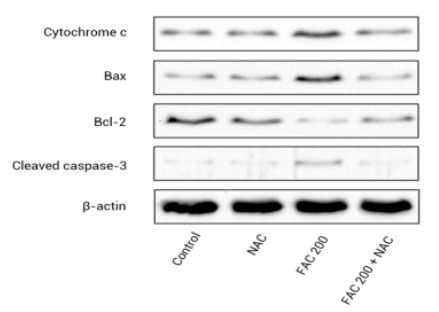

755

Figure 8 Protective effects of NAC against iron-induced apoptosis.

Osteoblasts were exposed to FAC $(200 \mu \mathrm{M})$ with or or without NAC $(1 \mathrm{mM})$ for $120 \mathrm{~h}$. (A) The change of intracellular ROS levels in osteoblasts treated with FAC $(200 \mu \mathrm{M})$ with or without NAC (1mM) for 120 h. $* \mathrm{P}<0.05$ vs the control ; $\# \mathrm{P}<0.05$ vs. FAC 200. (B) The change of MMP in 
osteoblasts incubated wtih FAC $(200 \mu \mathrm{M})$, in the absence or presence of $1 \mathrm{mM} \mathrm{NAC}$ for $120 \mathrm{~h}$, as assayed by flow cytometry. ${ }^{*} \mathrm{P}<0.05$ vs. the control ; \#P $<0.05$ vs. FAC 200 . (C) The effect of NAC on iron-induced cell apoptosis as assayed by flow cytometry analysis. ${ }^{*} \mathrm{P}<0.05$ vs. the control ; \#P $<$ 0.05 vs. FAC 200. (D) The effect of NAC on iron-induced morphological changes in cells as visualized by phase-contrast micrograph and Hoechst 33342 staining. (E) The effect of NAC on the expression of apoptosis-related proteins in iron-treated osteoblastic cells. 


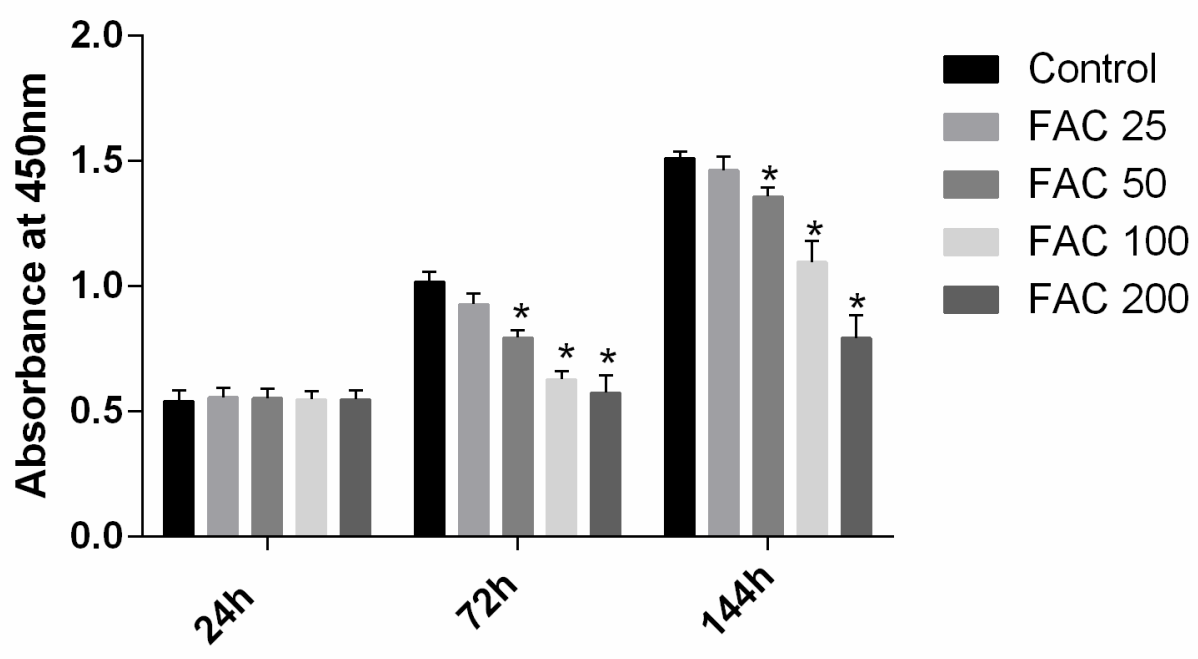



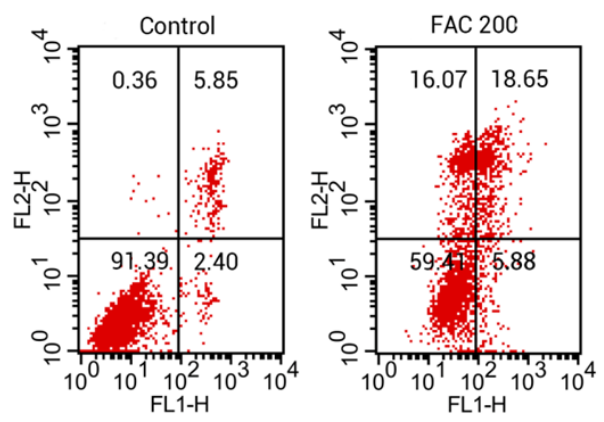

B

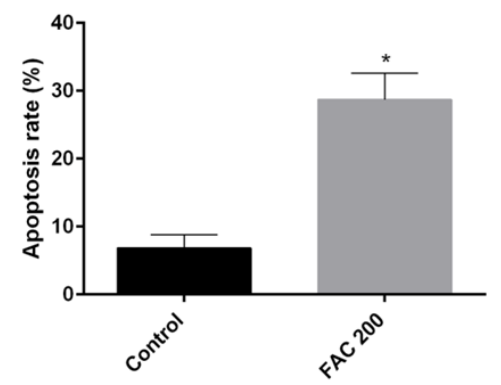
Figure 10 Iron induced apoptosis in bone marrow-derived MSCs. exposure to $200 \mu \mathrm{M}$ FAC for $144 \mathrm{~h}$. In each plot, the lower left quadrant represents live MSCs, the lower right and upper right quadrants represent apoptotic MSCs, and the upper left quadrant represents necrotic MSCs. (B) Statistical bar graphs show the mean values of flow cytometry data. Data are presented as the means $\pm \mathrm{SD}, \mathrm{n}=3 .{ }^{*} \mathrm{P}<0.05$ vs. the control. 
A

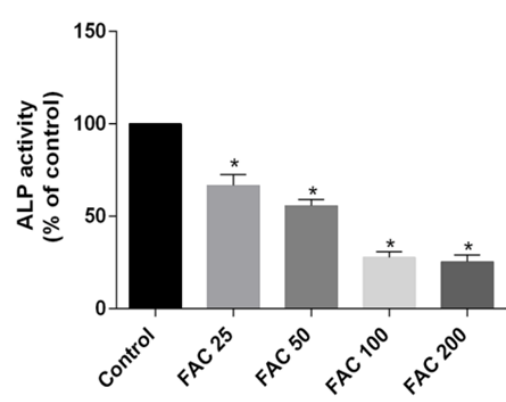

C

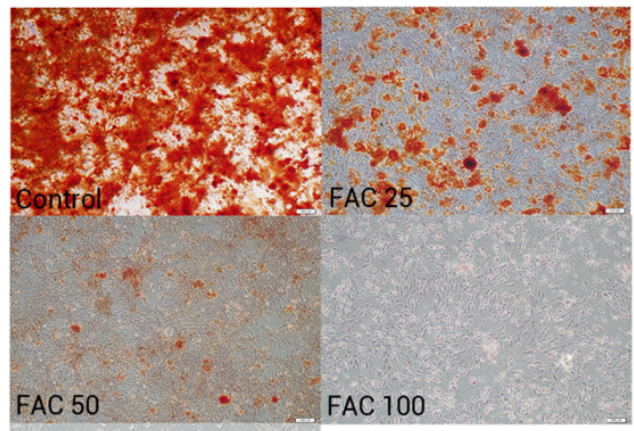

FAC 50
B

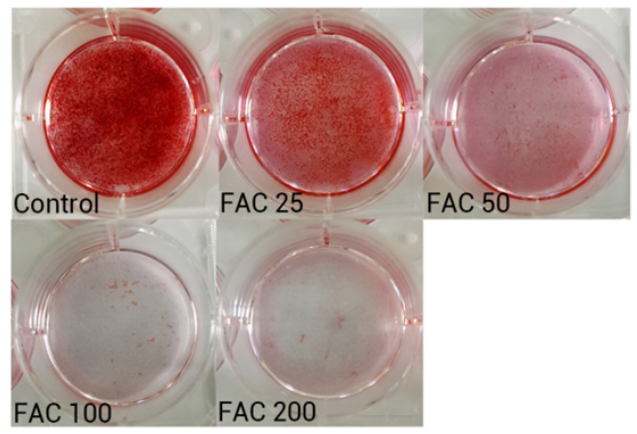

D

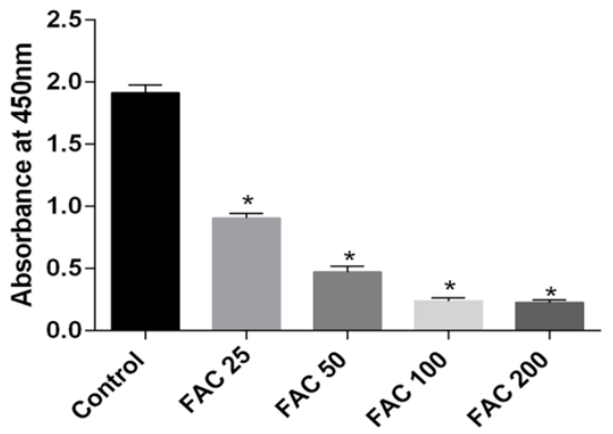

779

780

Figure 11 Effect of iron on ALP activity and matrix calcification

(A-D) Primary bone marrow-derived MSCs cultured in 6-well plates were induced with osteogenic medium supplemented with FAC $(25-200 \mu \mathrm{M})$ or alone for $14 \mathrm{~d}$. (A) ALP activity of MSCs was detected as described in Materials and Methods. Data are presented as the means $\pm \mathrm{SD}, \mathrm{n}=3$. ${ }^{*} \mathrm{P}<$ 0.05 vs. the control. (B-C) Mineralization in bone marrow-derived MSCs was assayed by Alizarin Red staining. Representative photographic images of stained wells (B) and microscopic views(C) are shown. (D) Statistical bar graphs show Ca content from different groups. Data are presented as the means $\pm \mathrm{SD}, \mathrm{n}=3 . * \mathrm{P}<0.05$ vs. the control. 


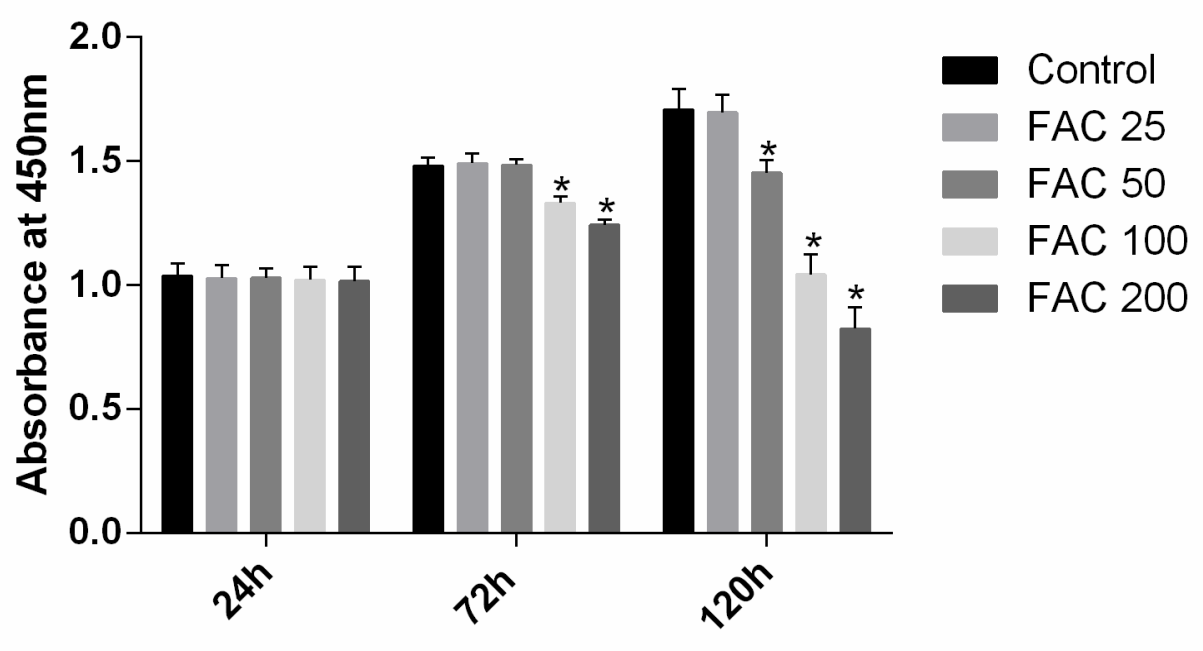
$* \mathrm{P}<0.05$ vs. the control. 
A

DUOX1

$\beta$-actin

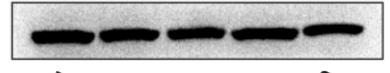

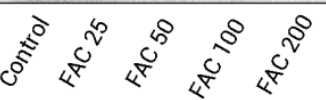

DUOX1

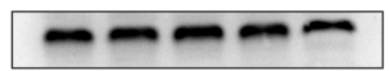

$\beta$-actin

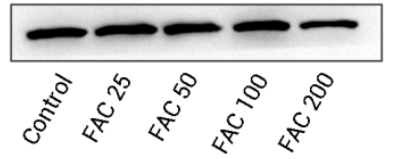

DUOX1

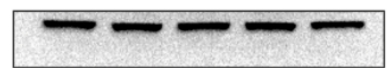

$\beta$-actin

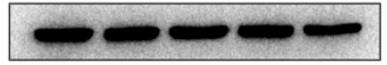

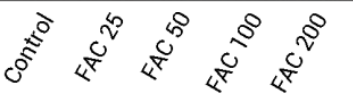

B

795

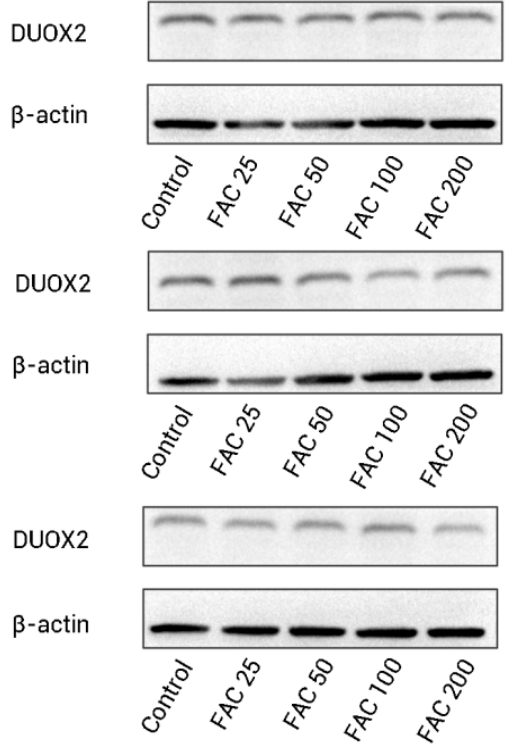
osteoblasts
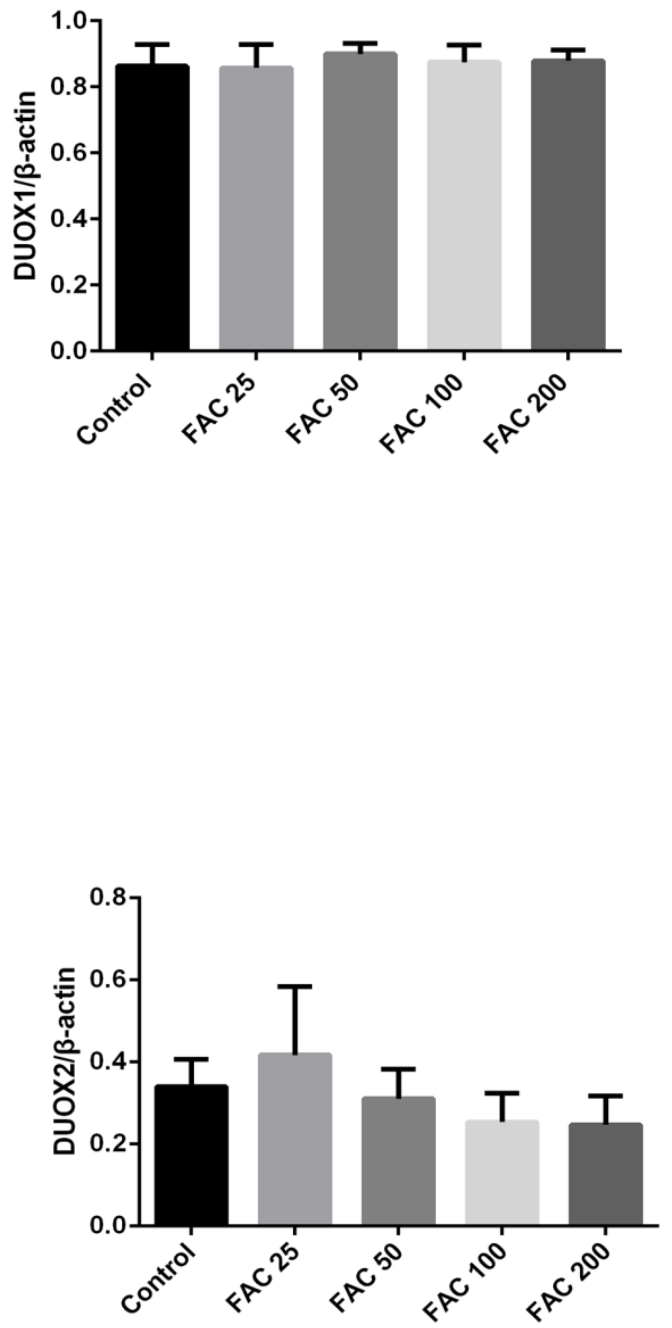

Supplementary Figure 2 Effect of iron on the exprssion of DUOX1 and DUOX2 in

(A-B) Representative western blot data for DUOX1 and DUOX2 in osteoblasts following exposure to $\operatorname{FAC}(0-200 \mu \mathrm{M})$ for $120 \mathrm{~h}$. $\beta$-actin was used as an internal control. Date are presented as means \pm 
$\mathrm{SD}, \mathrm{n}=3 ;{ }^{*} \mathrm{P}<0.05$ vs. the control. 